\title{
Purity for Barsotti-Tate groups in some mixed characteristic situations
}

\author{
Ofer Gabber and Adrian Vasiu
}

\begin{abstract}
Let $p$ be a prime. Let $R$ be a regular local ring of dimension $d \geqslant 2$ and residue characteristic $p$ whose completion is isomorphic to $C(k)\left[\left[x_{1}, \ldots, x_{d}\right]\right] /(h)$, with $C(k)$ a Cohen ring of the residue field $k$ of $R$ and with $h \in C(k)\left[\left[x_{1}, \ldots, x_{d}\right]\right]$ such that its reduction modulo $p$ does not belong to the ideal $\left(x_{1}^{p}, \ldots, x_{d}^{p}\right)+\left(x_{1}, \ldots, x_{d}\right)^{2 p-2}$ of $k\left[\left[x_{1}, \ldots, x_{d}\right]\right]$. We extend a result of Vasiu-Zink (for $d=2$ ) to show that each Barsotti-Tate group over $\operatorname{Frac}(R)$ which extends to every local ring of $\operatorname{Spec}(R)$ of dimension 1 extends uniquely to a Barsotti-Tate group over $R$. This result corrects in many cases several errors in the literature. As an application, we get that if $Y$ is a regular integral scheme such that the completion of each local ring of $Y$ of residue characteristic $p$ is a formal power series ring over a complete discrete valuation ring of absolute ramification index at most $p-1$, then each Barsotti-Tate group over the generic point of $Y$ which extends to every local ring of $Y$ of dimension 1 extends uniquely to a Barsotti-Tate group over $Y$.
\end{abstract}

\section{Introduction}

Let $R$ be a local noetherian $\operatorname{ring}$ with residue field $k$. Let $X:=\operatorname{Spec}(R)$, and let $U:=X \backslash \operatorname{Spec}(k)$ be the punctured spectrum of $R$. Let $p$ be a prime number. The notation $R, k, X, U$ and $p$ is fixed throughout this article.

We will abbreviate a Barsotti-Tate group (that is, a $p$-divisible group) to BT and a truncated Barsotti-Tate group of level $n \in \mathbb{N}^{*}$ to $\mathrm{BT}_{n}$. We recall from [VZ10, Definition 2] that in the case where $\operatorname{depth}(R) \geqslant 2$, the local ring $R$ is called $p$-quasi-healthy if each BT over $U$ extends to a BT over $X$; from [Gro68, Exposé III, Corollaire 3.5] applied to coherent sheaves defined by the structure sheaves of truncated BTs over $X$, we get that such an extension is unique up to unique isomorphism.

We recall that the first examples of BTs over $X$ are obtained by considering an abelian scheme $\mathcal{A}$ over $X$ and by taking a direct summand of the $\mathrm{BT} \mathcal{A}\left[p^{\infty}\right]$ of $\mathcal{A}$, that is, of the inductive system $\mathcal{A}\left[p^{n}\right]=\operatorname{Ker}\left(p^{n}: \mathcal{A} \rightarrow \mathcal{A}\right)$ indexed by $n \in \mathbb{N}^{*}$. If $R$ is complete and $k$ is an algebraically closed field of characteristic $p$, then the converse holds; that is, each BT over $X$

Received 20 February 2020, accepted in final form 27 January 2021.

2020 Mathematics Subject Classification 11G10, 11G18, 14F30, 14G35, 14K10, 14K99, 14L05, $14 \mathrm{~L} 15$.

Keywords: Barsotti-Tate groups, deformation theories, formal schemes, projective varieties, purity, regular rings, vector bundles.

This journal is (C) Foundation Compositio Mathematica 2021. This article is distributed with Open Access under the terms of the Creative Commons Attribution Non-Commercial License, which permits non-commercial reuse, distribution, and reproduction in any medium, provided that the original work is properly cited. For commercial re-use, please contact the Foundation Compositio Mathematica.

The second author was partially supported by the NSF grant DMS \#0900967. 


\section{Purity For BARsotti-TATE GROUPS}

is a direct summand of $\mathcal{A}\left[p^{\infty}\right]$ for some abelian scheme $\mathcal{A}$ over $X$ (this follows from [Vas06, Proposition 5.3.3]).

If $p=0$ in $R$ and $\operatorname{depth}(R) \geqslant 2$, then one can check that $R$ is not $p$-quasi-healthy using a variant of Moret-Bailly's example in [FC90, Chapter V, end of Section 6]. ${ }^{1}$

In [VZ10, Theorem 3], it is shown that there are large classes of $p$-quasi-healthy regular rings of dimension 2 and mixed characteristic $(0, p)$. This result already has applications to the uniqueness of integral canonical models of Shimura varieties and to the existence of new classes of Néron models (see [VZ10, Corollary 30 and Theorem 31]), to the study of moduli spaces of polarized K3 surfaces (see [Mad15, Mad16]), and to the study of Barsotti-Tate representations of fundamental groups of generic fibers of suitable formally smooth algebras over complete discrete valuation rings of absolute ramification index at most $p-2$ (see [LM20, Moo20]).

But no examples of $p$-quasi-healthy regular rings of mixed characteristic $(0, p)$ are (correctly) proved to exist in the literature for dimensions at least 3 ; this is so due to the fact that all claims in the literature for dimensions at least 3 rely on the erroneous argument in [FC90, Chapter V, Section 6 , reduction steps in the proof of Theorem $\left.6.4^{\prime}\right]$. The difficulty we encounter consists in being able to provide examples of dimension 3 as the Grothendieck-Messing deformation theory for BTs allows a relatively direct passage from dimension 3 to higher dimensions (see Lemma 2.8).

Our goal is to obtain purity results for BTs over regular schemes. More precisely, we will provide many examples of $p$-quasi-healthy regular local rings of dimension at least 3 , and we will use them to also get new examples of faithfully flat $\operatorname{Spec}\left(\mathbb{Z}_{(p)}\right)$-schemes which are $p$-healthy regular in the sense of either [Vas99, Definition 3.2.1 9)] or [VZ10, Definition 1]. Our main result is the following theorem, which extends [VZ10, Theorem 3].

Theorem 1.1. Let $R$ be a regular local ring of dimension $d \geqslant 1$ and mixed characteristic $(0, p)$ which satisfies the following condition:

(घ) The completion of $R$ is isomorphic to $C(k)\left[\left[x_{1}, \ldots, x_{d}\right]\right] /(h)$, with $C(k)$ a Cohen ring of $k$ and with $h \in C(k)\left[\left[x_{1}, \ldots, x_{d}\right]\right]$ such that its reduction $\bar{h}$ modulo $p$ does not belong to the ideal $\left(x_{1}^{p}, \ldots, x_{d}^{p}\right)+\left(x_{1}, \ldots, x_{d}\right)^{2 p-2}$ of $k\left[\left[x_{1}, \ldots, x_{d}\right]\right]$.

Then the following two properties hold:

(a) If a $\mathrm{BT}$ over $\operatorname{Frac}(R)$ extends to each local ring of $X=\operatorname{Spec}(R)$ of dimension 1 , then it extends uniquely (up to unique isomorphism) to a BT over $X$.

(b) If $d \geqslant 2$, then the regular local ring $R$ is p-quasi-healthy.

Clearly, statement (a) implies statement (b) and the fact that $X$ is also $p$-healthy regular. Condition $(\downarrow)$ is stable under generization; see Proposition 3.5. Based on this and on the classical purity theorem of Zariski, Nagata and Grothendieck (see [Gro68, Exposé X, Théorème 3.4(i)]), we get that, in fact, statements (a) and (b) are equivalent (see Section 7).

For $d=2$, Theorem 1.1(b) is proved in [VZ10, Theorem 3]. Theorem 1.1(b) and [VZ10, Proposition 23(b)] imply [VZ10, Theorem 3] and that $R$ for $d \geqslant 2$ is also a quasi-healthy regular

\footnotetext{
${ }^{1}$ We consider a homomorphism $\alpha_{\mathbf{p}, X} \rightarrow \alpha_{\mathbf{p}, X}^{\operatorname{dim}(R)}$ defined by a system of parameters of $R$ (it is a closed embedding over $U$ but not over $X$ ) and (as in a theorem of Raynaud) an embedding of $\alpha_{\mathbf{p}, X}^{\operatorname{dim}(R)}$ as a closed subgroup scheme of an abelian scheme $\mathcal{A}$ over $X$. If $\mathcal{D}_{U}:=\mathcal{A}_{U}\left[p^{\infty}\right] / \alpha_{\mathbf{p}, U}$ extends to a BT $\mathcal{D}$ over $X$, then by the depth assumption on $R$, the isogeny $\mathcal{A}_{U}\left[p^{\infty}\right] \rightarrow \mathcal{D}_{U}$ extends to a morphism $\mathcal{A}\left[p^{\infty}\right] \rightarrow \mathcal{D}$, which must be an isogeny (see [CCO14, Proposition 3.3.8 and the last part of Example 3.3.10]), so its kernel is a finite flat subgroup scheme of $\alpha_{\mathbf{p}, X}^{\operatorname{dim}(R)}$, which leads to a contradiction.
} 


\section{O. Gabber and A. Vasiu}

ring in the sense of [VZ10, Definition 2], which means that each abelian scheme over $U$ extends (uniquely up to a unique isomorphism) to an abelian scheme over $X .^{2}$ In this way, we get precisely those examples of quasi-healthy regular rings one gets based on only [VZ10, Theorem 3 and Lemma 24]. This is so because, based on Propositions 3.3 and 3.4, we easily get that for $d \geqslant 2$, the condition on $R$ in [VZ10, Theorem 3] is equivalent to ( $\downarrow$ ).

For $d \geqslant 3$, we prove Theorem 1.1(b) by induction on $d \geqslant 3$ (see Section 6), and the hard part is the case where $d=3$ (see Subsection 6.1). The proof of Theorem 1.1(b) for $d=3$ involves a study of the blowup of $X$ along its closed point $\operatorname{Spec}(k)$ and relies heavily on several important results and ideas.

Firstly, the proof of Theorem 1.1(b) for $d=3$ relies on an application (see Proposition 2.4) of Raynaud's complement to Tate's extension theorem [Tat67, Theorem 4] obtained in [Ray74, Proposition 2.3.1] and re-proved in Proposition 2.3 based on a refinement of [VZ12, Corollary 4] proved in Lemma 2.2. Secondly, the proof of Theorem 1.1(b) for $d=3$ relies on the case $d=2$ of Theorem 1.1(b) proved in [VZ10]. Thirdly, it relies on the particular case $\left(N, l^{\prime}, \mathcal{C}\right)=\left(2, l, \mathbb{P}_{l}^{1}\right)$ of the general theorem below, which we think is of interest in its own.

TheOREM 1.2. Let $l$ be a field of characteristic $p$. Let $N \in \mathbb{N}^{*}$ be an integer. For each $n \in \mathbb{N}^{*}$, let $\mathcal{D}_{n}$ be a $\mathrm{BT}_{n}$ over an open subscheme $\mathcal{U}_{n}$ of $\mathbb{P}_{l}^{N}$. We assume that we have a chain of inclusions $\mathcal{U}_{1} \supset \mathcal{U}_{2} \supset \cdots \supset \mathcal{U}_{n} \supset \cdots$ and that for all $n \in \mathbb{N}^{*}$, we have an identification $\mathcal{D}_{n+1}\left[p^{n}\right]=\mathcal{D}_{n, \mathcal{U}_{n+1}}$. We also assume that there exist a field extension $l^{\prime}$ of $l$ and a closed subscheme $\mathcal{C}$ of $\mathbb{P}_{l^{\prime}}^{N}$ of dimension greater than 0 and contained in $\mathcal{U}_{n, l^{\prime}}$ for all $n \in \mathbb{N}^{*}$ such that the inductive system $\mathcal{C} \times \mathcal{U}_{n} \mathcal{D}_{n}$ indexed by $n \in \mathbb{N}^{*}$ is a constant BT over $\mathcal{C}$; that is, it is isomorphic to the pullback to $\mathcal{C}$ of a BT over $\operatorname{Spec}\left(l^{\prime}\right)$. Then for each $n \in \mathbb{N}^{*}$, the $\mathrm{BT}_{n} \mathcal{D}_{n}$ extends uniquely (up to unique isomorphism) to a constant $\mathrm{BT}_{n} \mathcal{D}_{n}^{+}$over $\mathbb{P}_{l}^{N}$ isomorphic to $G\left[p^{n}\right]_{\mathbb{P}_{l}^{N}}$ for a suitable BT $G$ over $\operatorname{Spec}(l)$, and the identification $\mathcal{D}_{n+1}\left[p^{n}\right]=\mathcal{D}_{n, \mathcal{U}_{n+1}}$ extends to an identification $\mathcal{D}_{n+1}^{+}\left[p^{n}\right]=\mathcal{D}_{n}^{+}$ which is the pullback to $\mathbb{P}_{l}^{N}$ of the identification $G\left[p^{n+1}\right]\left[p^{n}\right]=G\left[p^{n}\right]$ over $\operatorname{Spec}(l)$. (Therefore, the BT over the stable under generization, pro-constructible subset $\mathcal{U}_{\infty}:=\cap_{n=1}^{\infty} \mathcal{U}_{n}$ of $\mathbb{P}_{l}^{N}$ induced naturally by the truncated $\mathrm{BTs} \mathcal{D}_{n}$ extends uniquely up to unique isomorphism to a constant BT over $\mathbb{P}_{l}^{N}$ isomorphic to $G_{\mathbb{P}_{l}^{N}}$ ).

Remark 1.3. Note that $\mathcal{U}_{\infty}$ is a locally ringed space, and one can define a BT over a locally ringed space $\left(\mathcal{Y}, \mathcal{O}_{\mathcal{Y}}\right)$ as a projective system of commutative and cocommutative Hopf $\mathcal{O}_{\mathcal{Y}}$-algebras $\mathcal{H}_{n}$ indexed by $n \in \mathbb{N}^{*}$, locally free of finite rank as $\mathcal{O}_{\mathcal{Y}}$-modules, such that for every point $z \in \mathcal{Y}$, the inductive system $\operatorname{Spec}\left(\left(\mathcal{H}_{n}\right)_{z}\right)$ of finite flat group schemes indexed by $n \in \mathbb{N}^{*}$ and defined by the stalks at $z$ constitutes a BT over the local scheme $\operatorname{Spec}\left(\mathcal{O}_{\mathcal{Y}, z}\right)$.

The proof of Theorem 1.2 is presented in Section 5, and it relies on properties of rings of formal-rational functions established in [HM68]. Theorem 1.1 is proved in Sections 6 and 7. Section 2 presents a few basic extension results that are required in the proof and the applications of Theorem 1.1, including a descent lemma for p-quasi-healthy regular rings (see Lemma 2.6) and the proof of Raynaud's complement to it. Section 3 groups a few basic properties related to condition ( $($ ) . Section 4 contains an elementary formal algebraic geometry property which is a standard application of the results of [HM68] and which plays a key role in the proof of Theorem 1.2.

The following corollary of Theorem 1.1 and its proof and Bondarko's boundedness results for truncated BTs over discrete valuation rings of mixed characteristic $(0, p)$ (see [Bon06] and [VZ12])

\footnotetext{
${ }^{2}$ It is an open problem when this extension property holds for polarized K3 surfaces.
} 


\section{PURITy FOR BARsotTi-TATE Groups}

extends [VZ10, Corollary 5], and it can be used to correct the argument used in [FC90, Chapter V, Section 6 , reduction steps in the proof of Theorem $\left.6.4^{\prime}\right]$ in many situations.

Corollary 1.4. Let $Y$ be a regular integral scheme flat over $\operatorname{Spec}(\mathbb{Z})$. We assume that for each point $z \in Y$ of characteristic $p$, condition $(\measuredangle)$ holds for the local ring $\mathcal{O}_{Y, z}$ of $z$ in $Y$; this holds if the completion of $\mathcal{O}_{Y, z}$ is formally smooth (for example, is a formal power series ring) over a complete discrete valuation ring of absolute ramification index $e \in\{1, \ldots, p-1\}$. Then the following two properties hold:

(a) Each BT over the generic point of $Y$ which extends to every local ring of $Y$ of dimension 1 extends uniquely (up to unique isomorphism) to a BT over $Y$.

(b) Each BT over an open subscheme of $Y$ that contains $Y[1 / p]$ and all points of $Y_{\mathbb{F}_{p}}$ of codimension 0 in $Y_{\mathbb{F}_{p}}$ extends to a BT over $Y$ (thus, if $Y$ is a faithfully flat $\operatorname{Spec}\left(\mathbb{Z}_{(p)}\right)$-scheme, then it is p-healthy regular).

[We clarify that in Corollary 1.4, a regular scheme means a scheme whose local rings are noetherian and regular, but it is not assumed to be locally noetherian; the boundedness results are necessary to treat the non-locally noetherian case.]

The case $e<p-1$ of Corollary 1.4(b) is a special case of [FC90, Chapter V, Theorem 6.4'] (which is incorrect already in dimension 2) and was claimed more recently in [Moo98, Section 3.6.1] and in [Vas99, Remark 3.2.2 3) and the last two lines of Section 3.2.17]. The case $e=1$ of Corollary 1.4(b) was also claimed in [Vas04, Theorem 1.3]. Unfortunately, the inductive passages from dimension 2 to dimension 3 in all four of these references relied on [FC90, Chapter V, Section 6, argument on pp. 183-184], which, in the language of this paper, would in particular imply that for each integer $n \geqslant 2$, all local noetherian rings $C(k)\left[\left[x_{1}, x_{2}\right]\right] /\left(x_{2}^{n}\right)$ are $p$-quasi-healthy. The counterexample of [VZ10, Section 5.1] shows that the last statement is false.

The hypothesis of Corollary 1.4(a) holds for each BT over the generic point of $Y$ whose truncations extend to finite flat group schemes over $Y$ (Raynaud's extension theorem). Thus Corollary 1.4(a) reobtains, refines and generalizes the first part of [Moo20, Theorem 1.2], which worked in certain formally smooth contexts over a discrete valuation ring of absolute ramification index $e<p-1$.

If $Y$ is as in Corollary 1.4 and is faithfully flat over $\operatorname{Spec}\left(\mathbb{Z}_{(p)}\right)$, then $Y$ is also a healthy regular scheme in the sense of [Vas99, Definition 3.2.1 2)]; that is, each abelian scheme over an open subscheme of $Y$ that contains $Y_{\mathbb{Q}}$ and all points of $Y_{\mathbb{F}_{p}}$ of codimension 0 in $Y_{\mathbb{F}_{p}}$ extends to an abelian scheme over $Y$ (see [Vas04, Proposition 4.1]). From this and [VZ10, Lemma 29], one gets a second proof of the uniqueness of integral canonical models of Shimura varieties defined in [Vas99, Definition 3.2.3 6)] over discrete valuation rings of mixed characteristic $(0, p)$ and absolute ramification index at most $p-1$, which was first proved in [VZ10, Corollary 30].

The examples of [VZ10, Theorem 28(i) and (ii)] for $d=2$ can easily be adapted to provide many examples of regular local rings $R$ of arbitrary dimension $d \geqslant 2$ which are of mixed characteristic $(0, p)$ and are not $p$-quasi-healthy. For example, the regular complete local rings $C(k)\left[\left[x_{1}, \ldots, x_{d}\right]\right] /\left(p-a x_{1}^{p}\right)$ and $C(k)\left[\left[x_{1}, \ldots, x_{d}\right]\right] /\left(p-a \prod_{i=1}^{d} x_{i}^{p-1}\right)$, where $a \in C(k)\left[\left[x_{1}, \ldots, x_{d}\right]\right]$, are not $p$-quasi-healthy; in particular, formal power series rings in $d-1 \geqslant 1$ indeterminates over a complete discrete valuation ring of absolute ramification index at least $p$ are not $p$-quasi-healthy. See [GV20] for details, generalizations and the fact that if $R$ is regular henselian of dimension 2, then condition ( $\bullet$ ) holds if and only if $R$ is $p$-quasi-healthy.

Complements to Corollary 1.4 and to Lemma 7.1 used in its proof are included in Section 8. 


\section{O. Gabber and A. Vasiu}

Let $\mathcal{O}_{b}$ be the structure sheaf of a scheme $b$. For each local noetherian ring $R$, let $\widehat{R}$ be its completion. If $R$ is an integral domain, let $K:=\operatorname{Frac}(R)$; so if $R$ is a discrete valuation ring, then $U=\operatorname{Spec}(K)$. If $k$ is perfect of characteristic $p$, then $C(k)$ is the ring $W(k)$ of $p$-typical Witt vectors with coefficients in $k$. We think of a BT over a scheme as an inductive system indexed by elements of $\mathbb{N}^{*}$ or alternatively as its limit fppf sheaf; see [CCO14, Section 3.3.2].

\section{Basic extension properties}

In this section, we gather some basic extension properties of a different nature that will be used often in what follows. We begin by recalling some standard properties of reflexive sheaves.

\subsection{Reflexive sheaves}

We will often use the following elementary fact.

FACT 2.1. Let $\mathcal{N}$ be a normal noetherian scheme. Let $\mathcal{U}$ be an open dense subscheme of $\mathcal{N}$ which contains all codimension 1 points (that is, points whose local rings are discrete valuation rings). Let $\mathcal{E}$ and $\mathcal{H}$ be two finite flat group schemes over $\mathcal{N}$. Let $\mathcal{V}$ be a reflexive coherent $\mathcal{O}_{\mathcal{N}}$-module. Then the following four properties hold:

(a) If $\mathcal{N}$ is regular of dimension at most 2, then the restriction from $\mathcal{N}$ to $\mathcal{U}$ induces an equivalence of categories from the category of coherent locally free $\mathcal{O}_{\mathcal{N}}$-modules to the category of coherent locally free $\mathcal{O}_{\mathcal{U}}$-modules.

(b) If $\mathcal{N}$ is regular of dimension at most 2, then the restriction from $\mathcal{N}$ to $\mathcal{U}$ induces an equivalence of categories from the category of finite flat group schemes over $\mathcal{N}$ to the category of finite flat group schemes over $\mathcal{U}$.

(c) The restriction homomorphisms $\operatorname{Hom}(\mathcal{E}, \mathcal{H}) \rightarrow \operatorname{Hom}\left(\mathcal{U} \times_{\mathcal{N}} \mathcal{E}, \mathcal{U} \times_{\mathcal{N}} \mathcal{H}\right)$ and $H^{0}(\mathcal{N}, \mathcal{V}) \rightarrow$ $H^{0}(\mathcal{U}, \mathcal{V})$ are isomorphisms.

(d) Let $f: \mathcal{E} \rightarrow \mathcal{H}$ be a homomorphism. Let $\mathcal{W}$ be the largest open subscheme of $\mathcal{N}$ such that the restriction $f_{\mathcal{W}}: \mathcal{E}_{\mathcal{W}} \rightarrow \mathcal{H}_{\mathcal{W}}$ of $f$ to a homomorphism over $\mathcal{W}$ is an isomorphism. Then $\mathcal{W}$ is the set of all points $z \in \mathcal{N}$ at which the fiber $f_{z}$ of $f$ is an isomorphism. Moreover, $\mathcal{W}$ contains all points of codimension at most 1 in $\mathcal{N}$ if and only if we have $\mathcal{W}=\mathcal{N}$ (that is, $f$ is an isomorphism if and only if its fibers over points of codimension at most 1 in $\mathcal{N}$ are isomorphisms).

For statement (a), one uses extension of coherent sheaves and [Har80, Corollary 1.4], and for the second part of statement (c), we refer to [Har80, Proposition 1.6].

\subsection{A refinement of a result of [VZ12]}

In this subsection, we assume that $R$ is a discrete valuation ring of mixed characteristic $(0, p)$. Thus $K=\operatorname{Frac}(R)$ and $U=\operatorname{Spec}(K)$. Let $s \in \mathbb{N}$ be such that [VZ12, Theorem 1] holds for $R$; it has upper bounds in terms of only the absolute ramification index $e$ of $R$ (the best upper bound $s=\left[\log _{p}(p e /(p-1))\right]$ is obtained in [Bon06, Theorem A]). If $H$ is a finite flat commutative group scheme of $p$-power order over $X$, then for $n \in \mathbb{N}$, let $H\left\lceil p^{n}\right\rceil$ be the schematic closure of $H_{K}\left[p^{n}\right]$ in $H$; so $H\left\lceil p^{0}\right\rceil=H\lceil 1\rceil$ is the trivial subgroup scheme of $H$.

The following lemma refines [VZ12, Corollary 4], which worked in a context in which $H^{\prime}$ is a $\mathrm{BT}_{n}$ over $X$. 


\section{Purity For BARsotti-TATE GROUPS}

Lemma 2.2. With $R, K$ and $s$ as above, let $n>2 s$ be an integer. Let $H$ and $H^{\prime}$ be finite flat group schemes over $X$ such that their generic fibers $H_{K}$ and $H_{K}^{\prime}$ are $\mathrm{BT}_{n} s$ over $U=\operatorname{Spec}(K)$. For $i \in\{0, \ldots, n\}$, let $H_{i}:=H\left\lceil p^{i}\right\rceil$ and $H_{i}^{\prime}:=H^{\prime}\left\lceil p^{i}\right\rceil$. Then the following three properties hold:

(a) If $f: H \rightarrow H^{\prime}$ is a homomorphism such that $f_{K}: H_{K} \rightarrow H_{K}^{\prime}$ is an isomorphism, then the homomorphism $H_{n-s} / H_{s} \rightarrow H_{n-s}^{\prime} / H_{s}^{\prime}$ induced by $f$ is an isomorphism.

(b) If $H_{K} \rightarrow H_{K}^{\prime}$ is a homomorphism (respectively, is an isomorphism), then the homomorphism $H_{K}\left[p^{n-s}\right] / H_{K}\left[p^{s}\right] \rightarrow H_{K}^{\prime}\left[p^{n-s}\right] / H_{K}^{\prime}\left[p^{s}\right]$ induced by it extends to a homomorphism (respectively, to an isomorphism) $H_{n-s} / H_{s} \rightarrow H_{n-s}^{\prime} / H_{s}^{\prime}$.

(c) If $n \geqslant 2 s+2$, then $H_{s+t} / H_{s}$ is a $\mathrm{BT}_{t}$ over $X$ for all $t \in\{1, \ldots, n-2 s\}$.

Proof. For $i \in\{0, \ldots, n-1\}$, let $\operatorname{gr}_{i+1}(H):=H_{i+1} / H_{i}$ and $\operatorname{gr}_{i+1}\left(H^{\prime}\right):=H_{i+1}^{\prime} / H_{i}^{\prime}$. To prove statement (a), we consider the commutative diagram

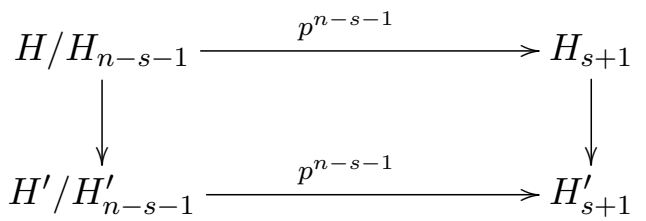

whose vertical arrows are induced by $f$. Let $\alpha: H / H_{n-s-1} \rightarrow H_{s+1}^{\prime}$ be the diagonal homomorphism of the diagram, and let $\beta: H_{s+1}^{\prime} \rightarrow H / H_{n-s-1}$ be a homomorphism such that $\alpha \circ \beta$ and $\beta \circ \alpha$ are both multiplication by $p^{s}$ (which exists by our choice of $s$ ). Then $p^{s} \beta$ induces a homomorphism $\operatorname{gr}_{s+1}\left(H^{\prime}\right) \rightarrow \operatorname{gr}_{n-s}(H)$ which is an inverse of the homomorphism $\operatorname{gr}_{n-s}(H) \rightarrow \operatorname{gr}_{s+1}\left(H^{\prime}\right)$ induced by $p^{n-2 s-1} f$. Using factorizations of the isomorphism $\operatorname{gr}_{n-s}(H) \rightarrow \operatorname{gr}_{s+1}\left(H^{\prime}\right)$, we get that for $i \in\{s+1, \ldots, n-s\}$, the homomorphisms $\operatorname{gr}_{i+1}(H) \rightarrow \operatorname{gr}_{i+1}\left(H^{\prime}\right)$ induced by $f$ are isomorphisms. This implies that statement (a) holds.

In the situations of statement (b), consider the schematic closure $\tilde{H}$ of the graph of $H_{K} \rightarrow H_{K}^{\prime}$ in the product $H \times_{\operatorname{Spec}(R)} H^{\prime}$. The projections $\tilde{H} \rightarrow H$ and $\tilde{H} \rightarrow H^{\prime}$ induce homomorphisms $\tilde{\alpha}: \tilde{H}_{n-s} / \tilde{H}_{s} \rightarrow H_{n-s} / H_{s}$ and $\tilde{\beta}: \tilde{H}_{n-s} / \tilde{H}_{s} \rightarrow H_{n-s}^{\prime} / H_{s}^{\prime}$. By statement (a), the homomorphism $\tilde{\alpha}$ is an isomorphism (respectively, $\tilde{\alpha}$ and $\tilde{\beta}$ are isomorphisms), which implies the assertions.

To prove statement (c), from the proof of statement (a) applied to the identity automorphism of $H$, we get that the homomorphism $\operatorname{gr}_{n-s}(H) \rightarrow \operatorname{gr}_{s+1}(H)$ is an isomorphism, and this implies that for each $i \in\{s+1, \ldots, n-s-1\}$, the successive homomorphism $\operatorname{gr}_{i+1}(H) \rightarrow \operatorname{gr}_{i}(H)$ (which is induced by the multiplication by $p$ and is an isomorphism over $U$ ) is an isomorphism. Thus $H_{n-s} / H_{s}$ is an fppf sheaf that is flat over $\mathbb{Z} / p^{n-2 s} \mathbb{Z}$. From [Mes72, Chapter 1, Definition 1.2], we get that statement (c) holds for $t=n-2 s$. If $1 \leqslant t \leqslant n-2 s-1$, then $\left(H_{n-s} / H_{s}\right)\left[p^{t}\right]$ is a $\mathrm{BT}_{t}$ over $X$ and is equal to the flat closed subgroup scheme $H_{s+t} / H_{s}$ of $H_{n-s} / H_{s}$ as this is so over $U$.

\subsection{On Raynaud's complement to Tate's extension theorem}

We recall Raynaud's complement to Tate's extension theorem (see [Ray74, Proposition 2.3.1]) and include a new proof of it based on Lemma 2.2.

Proposition 2.3. We assume that $R$ is a discrete valuation ring of mixed characteristic $(0, p)$. Let $D_{K}$ be a BT over $K=\operatorname{Frac}(R)$. If for each $n \in \mathbb{N}^{*}$, the $\mathrm{BT}_{n} D_{K}\left[p^{n}\right]$ extends to a finite flat group scheme $E_{n}$ over $X$, then $D_{K}$ extends to a BT over $X$.

Proof. If $t \geqslant 2$ is an integer, then we have $2 s+t \geqslant 2 s+2$ and the quotient group scheme $F_{t}:=E_{2 s+t}\left\lceil p^{s+t}\right\rceil / E_{2 s+t}\left\lceil p^{s}\right\rceil$ is a $\mathrm{BT}_{t}$ which extends $D_{K}\left[p^{t}\right]$ and does not depend on $E_{2 s+t}$ 


\section{O. Gabber and A. Vasiu}

(see Lemma 2.2(b) and (c)). Taking $E_{2 s+t}=E_{2 s+t+1}\left\lceil p^{2 s+t}\right\rceil$, we get that $F_{t}=F_{t+1}\left[p^{t}\right]$. Thus $F_{1}:=F_{t}[p]$ does not depend on $t$, and the inductive system $F_{m}$ indexed by $m \in \mathbb{N}^{*}$ is a BT over $X$ that extends $D_{K}$.

In the proof of Theorem 1.1(b), we will need the following application of Proposition 2.3.

Proposition 2.4. Let $R$ be a regular local ring of mixed characteristic $(0, p)$ and dimension $d \geqslant 2$. Let $D_{U}$ be a BT over $U$ (the punctured spectrum of $R$ ). Let $O$ be the discrete valuation ring which dominates $R$, which has the same field of fractions $K$ as $R$ and which is a local ring of the blowup of $X$ along its closed point (thus the residue field of $O$ is a field of rational functions in $d-1$ indeterminates over $k$ ). Then the generic fiber $D_{K}$ of $D_{U}$ over $\operatorname{Spec}(K)$ extends uniquely (up to unique isomorphism) to a $\mathrm{BT}$ over $\operatorname{Spec}(O)$.

Proof. For each $n \in \mathbb{N}^{*}$, from Lemma 2.5 below applied with $\left(H_{K}, H_{U}\right)=\left(D_{K}\left[p^{n}\right], D_{U}\left[p^{n}\right]\right)$ we get that the $\mathrm{BT}_{n} D_{K}\left[p^{n}\right]$ over $\operatorname{Spec}(K)$ extends to a finite flat group scheme over $\operatorname{Spec}(O)$. Based on this, the proposition follows from Proposition 2.3 applied over $O$.

Lemma 2.5. Let $R, d, U, O, K$ and $X$ be as in Proposition 2.4. Let $H_{K}$ be a finite group scheme over $\operatorname{Spec}(K)$ which extends to a finite flat group scheme $H_{U}$ over $U$. Then $H_{K}$ extends to a finite flat group scheme over $\operatorname{Spec}(O)$.

Proof. Let $R_{1}$ be a regular local ring of dimension 2 which dominates $R$, which is dominated by $O$ and whose punctured spectrum $U_{1}$ is $X_{1} \times{ }_{X} U$, where $X_{1}:=\operatorname{Spec}\left(R_{1}\right)$. For instance, we can take $R_{1}$ to be a local ring of the blowup of $X$ along a regular closed subscheme of it of dimension 1 . From Fact 2.1(b), we get that $U_{1} \times_{U} H_{U}$ extends uniquely (up to unique isomorphism) to a finite flat group scheme $H_{1}$ over $X_{1}$. The pullback of $H_{1}$ to $\operatorname{Spec}(O)$ is a finite flat group scheme over $\operatorname{Spec}(O)$ that extends $H_{K}$.

\subsection{A descent lemma}

In what follows, we will often use the following general descent lemma.

Lemma 2.6. Let $R \rightarrow R^{\prime}$ be a faithfully flat extension between noetherian local rings of the same dimension. If depth $(R) \geqslant 2$ and $R^{\prime}$ is p-quasi-healthy, then $R$ is p-quasi-healthy.

Proof. Let $D_{U}$ be a BT over $U$. We have to show that $D_{U}$ extends (automatically uniquely up to unique isomorphism) to a BT $D$ over $X=\operatorname{Spec}(R)$. Let $X^{\prime}:=\operatorname{Spec}\left(R^{\prime}\right)$, and let $f: X^{\prime} \rightarrow X$ be the morphism induced by the monomorphism $R \rightarrow R^{\prime}$. From [Mat89, Theorem 15.1], we get that the closed fiber of $f$ has dimension 0 , and therefore $U^{\prime}:=f^{-1}(U)$ is the punctured spectrum of $R^{\prime}$. Thus $D_{U^{\prime}}=U^{\prime} \times_{U} D_{U}$ extends to a BT $D_{X^{\prime}}$ over $X^{\prime}$.

For $n \in \mathbb{N}^{*}$, let $A_{n}:=H^{0}\left(U, \mathcal{O}_{D_{U}\left[p^{n}\right]}\right)$. We have to show that the following two properties hold:

(i) For each $n \in \mathbb{N}^{*}$, the $R$-module $A_{n}$ is finitely generated projective (so for $t \in \mathbb{N}$, the $t$ fold tensor power of $A_{n}$ over $R$ maps isomorphically to $\mathcal{O}\left(D_{U}\left[p^{n}\right]^{t}\right)$ ), and thus the group scheme structure of $D_{U}\left[p^{n}\right]$ endows $A_{n}$ with a structure of commutative and cocommutative Hopf $R$-algebra that defines a finite flat commutative group scheme $D_{n}$ over $X$ that extends $D_{U}\left[p^{n}\right]$.

(ii) For all $n, m \in \mathbb{N}^{*}$, the complex $0 \rightarrow D_{m} \rightarrow D_{n+m} \rightarrow D_{n} \rightarrow 0$ that extends the short exact sequence $0 \rightarrow D_{U}\left[p^{m}\right] \rightarrow D_{U}\left[p^{n+m}\right] \rightarrow D_{U}\left[p^{n}\right] \rightarrow 0$ is a short exact sequence of commutative finite flat group schemes over $X$. 


\section{Purity For BARsotti-TATE GROUPS}

If these two properties hold, then we can take $D$ to be the inductive system $D_{n}$ (indexed by $n \in \mathbb{N}^{*}$ ). To check that properties (i) and (ii) hold, we can work locally in the fpqc topology, and thus these two properties follow from the fact that for each $n \in \mathbb{N}^{*}$, we have $D_{X^{\prime}}\left[p^{n}\right]=$ $\operatorname{Spec}\left(R^{\prime} \otimes_{R} A_{n}\right)=X^{\prime} \times_{X} D_{n}$.

\subsection{Extending vector bundles}

The following criterion for extending vector bundles will be used repeatedly in connection to extending truncated BTs.

LEMma 2.7. Let $R$ be a local noetherian ring of residue characteristic $p$ with $\operatorname{depth}(R) \geqslant 3$ and with a non-unit $y$ such that $(y, p)$ is a regular sequence of $R$ (so $R$ is of mixed characteristic $(0, p)$ ). Let $S$ be either $R$ or $R /\left(y^{n}\right)$ for some $n \in \mathbb{N}^{*}$. Let $\mathcal{F}$ be a vector bundle over the punctured spectrum $U_{S}$ of $S$. If $S=R$, let $\tau$ be $y$, and if $S=R /\left(y^{n}\right)$, let $\tau$ be $p$. For each $t \in \mathbb{N}^{*}$, let $\iota_{t}: U_{S} \cap \operatorname{Spec}\left(S /\left(\tau^{t}\right)\right) \rightarrow \operatorname{Spec}\left(S /\left(\tau^{t}\right)\right)$ be the restriction modulo $\tau^{t}$ of the open embedding $\iota: U_{S} \rightarrow \operatorname{Spec}(S)$. We assume that the following two conditions hold:

(a) For each $t \in \mathbb{N}^{*}$, the direct image $\iota_{t, *} \mathcal{F}_{t}$ of the restriction $\mathcal{F}_{t}$ of $\mathcal{F}$ to $U_{S} \cap \operatorname{Spec}\left(S /\left(\tau^{t}\right)\right)$ is a vector bundle $\mathcal{F}_{t}^{+}$over $\operatorname{Spec}\left(S /\left(\tau^{t}\right)\right)$.

(b) If $S=R /\left(y^{n}\right)$, then $\operatorname{depth}(R) \geqslant 4$.

Then the direct image $\iota_{*} \mathcal{F}$ is a vector bundle over $\operatorname{Spec}(S)$ whose restriction to $\operatorname{Spec}\left(S /\left(\tau^{t}\right)\right)$ maps isomorphically to $\mathcal{F}_{t}^{+}$for all $t \in \mathbb{N}^{*}$.

Proof. If $S=R$ is regular, then the lemma is a particular case of [FC90, Chapter V, Section 6, Lemma 6.6] applied over $\widehat{S}$. If $S=R$ (respectively, if $S=R /\left(y^{n}\right)$ ), then from the AuslanderBuchsbaum formula, we get that $\operatorname{depth}(S)$ is $\operatorname{depth}(R)-1 \geqslant 2(\operatorname{respectively,} \operatorname{depth}(R)-2 \geqslant 2)$. Thus, regardless of what $S$ is, the lemma is a particular case of [Gro68, Exposé IX, Proposition 1.4 and Exemple 1.5] applied over $\widehat{S}$.

\subsection{Extending BTs}

The next lemma is a natural application of Lemma 2.7 and is the essence of the inductive step of the induction we will use to prove Theorem 1.1(b).

Lemma 2.8. Let $R$ be a local noetherian ring of residue characteristic $p$ with $\operatorname{depth}(R) \geqslant 4$ and with a non-unit $y$ such that $(y, p)$ is a regular sequence of $R$. Let $S:=R /(y)$. Let $D_{U}$ be a BT over the punctured spectrum $U$ of $R$. If the restriction of $D_{U}$ to the punctured spectrum of $S$ extends to $\operatorname{Spec}(S)$, then $D_{U}$ extends to a $\mathrm{BT}$ over $X=\operatorname{Spec}(R)$.

Proof. Based on the proof of Lemma 2.6, we can assume that $R$ is complete. For $t \in \mathbb{N}^{*}$, let

$$
X_{t}:=\operatorname{Spec}\left(R /(y)^{t}\right)
$$

and $U_{t}:=U \cap X_{t}$. By induction on $t \in \mathbb{N}^{*}$, we will show that the restriction $D_{U_{t}}$ of $D_{U}$ to $U_{t}$ extends uniquely (up to unique isomorphism) to a BT $D_{X_{t}}$ over $X_{t}$. We already know that this holds for $t=1$. Let $\iota_{U_{1}}: D_{U_{1}} \rightarrow U_{1} \times_{X_{1}} D_{X_{1}}$ be the canonical isomorphism.

The passage from $t$ to $t+1$ goes as follows. Assuming that $D_{X_{t}}$ exists, let

$$
\iota_{U_{t}}: D_{U_{t}} \rightarrow U_{t} \times_{X_{t}} D_{X_{t}}
$$

be the canonical isomorphism. For $q \in \mathbb{N}^{*}$, let $D_{X_{t}, q}$ and $D_{U_{t}, q}$ be the reductions modulo $p^{q}$ of $D_{X_{t}}$ and $D_{U_{t}}$, respectively; they are BTs over the reductions $X_{t, q}$ and $U_{t, q}$ of $X_{t}$ and $U_{t}$, respectively, 


\section{O. Gabber and A. Vasiu}

modulo $p^{q}$. From the Grothendieck-Messing deformation theory (see [Ill85, Théorème 4.4 and Corollaire 4.7] and [Mes72, Chapter V, Theorem 1.6]), we get that the lifts of $D_{X_{t}, q}$ to BTs over $X_{t+1, q}$ are parametrized by the global sections of a torsor under the group of global sections of a coherent locally free $\mathcal{O}_{\operatorname{Spec}\left(S / p^{q} S\right)}$-module $\mathcal{F}_{t, q}$ (note that the ideal that defines the closed embedding $X_{t, q} \rightarrow X_{t+1, q}$ has a canonical trivial divided power structure, and therefore this closed embedding has a canonical structure of nilpotent divided power thickening). Similarly, the lifts of $D_{U_{t}, q}$ to BTs over $U_{t+1, q}$ are parametrized by a torsor under the group $H^{0}\left(U \cap \operatorname{Spec}\left(S / p^{q} S\right), \mathcal{F}_{t, q}\right)$. As $(y, p)$ is a regular sequence of $R$, we have $\operatorname{depth}\left(S / p^{q} S\right)=\operatorname{depth}(R)-2 \geqslant 2$, and thus we can identify $H^{0}\left(U \cap \operatorname{Spec}\left(S / p^{q} S\right), \mathcal{F}_{t, q}\right)=H^{0}\left(\operatorname{Spec}\left(S / p^{q} S\right), \mathcal{F}_{t, q}\right)$. This implies that there exists a unique (up to unique isomorphism) BT $D_{X_{t+1}, q}$ over $X_{t+1, q}$ that lifts compatibly both $D_{X_{t}, q}$ and $D_{U_{t+1}, q}$. As $R /(y)^{t+1}$ is $p$-adically complete, there exists a unique (up to unique isomorphism) BT $D_{X_{t+1}}$ over $X_{t+1}$ which lifts the system $D_{X_{t+1}, q}, q \in \mathbb{N}^{*}$ (see [Mes72, Chapter II, Lemma 4.16], which implies that the categories of BTs over $X_{t+1}$ and over the formal scheme which is the $p$-adic completion of $X_{t+1}$ are canonically equivalent).

For each $n \in \mathbb{N}^{*}$, from Lemma 2.7 applied with

$$
\left(S, \tau,\left(\mathcal{F}_{q}, \mathcal{F}_{q}^{+}\right)_{q \in \mathbb{N}^{*}}\right)=\left(R /(y)^{t+1}, p,\left(\mathcal{O}_{D_{U_{t+1}, q}\left[p^{n}\right]}, \mathcal{O}_{D_{X_{t+1}, q}\left[p^{n}\right]}\right)_{q \in \mathbb{N}^{*}}\right)
$$

we get that $\mathcal{O}_{D_{U_{t+1}}\left[p^{n}\right]}$ is the restriction to $U_{t+1}$ of a vector bundle over $X_{t+1}$ whose restriction to $X_{t+1, q}$ is compatibly identified with $\mathcal{O}_{D_{X_{t+1}, q}\left[p^{n}\right]}$ for all $q \in \mathbb{N}^{*}$, and thus, as $X_{t+1}$ is $p$ adically complete, this vector bundle can be functorially identified with $\mathcal{O}_{D_{X_{t+1}}\left[p^{n}\right]}$. We deduce an isomorphism $\iota_{U_{t+1}}\left[p^{n}\right]: D_{U_{t+1}}\left[p^{n}\right] \rightarrow U_{t+1} \times_{X_{t+1}} D_{X_{t+1}}\left[p^{n}\right]$ of $U_{t+1}$-schemes, and this must be a group scheme isomorphism. The isomorphisms $\iota_{U_{t+1}}\left[p^{n}\right]$ glue together to define an isomorphism

$$
\iota_{U_{t+1}}: D_{U_{t+1}} \rightarrow U_{t+1} \times_{X_{t+1}} D_{X_{t+1}} .
$$

Therefore, the restriction of $D_{X_{t+1}}$ to $U_{t+1}$ is canonically identified with $D_{U_{t+1}}$. This ends the induction.

From Lemma 2.7 applied with $(S, \tau)=(R, y)$, we similarly get that for each $n \in \mathbb{N}^{*}$, the locally free $\mathcal{O}_{U}$-module associated to $D_{U}\left[p^{n}\right]$ extends to a locally free $\mathcal{O}_{X}$-module whose reduction modulo each $(y)^{t}$ is the $\mathcal{O}_{X_{t}}$-module associated to $D_{X_{t}}\left[p^{n}\right]$ and which defines naturally a $\mathrm{BT}_{n} D_{n}$ over $X$. The inductive system $D_{n}$ is a BT over $X$ which extends $D_{U}$ (and thus our notation matches); we note that it is also the unique (up to unique isomorphism) BT over $X$ which lifts compatibly each $D_{X_{t}}$ with $t \in \mathbb{N}^{*}$ (see again [Mes72, Chapter II, Lemma 4.16]). Thus the lemma holds.

\section{On condition ( $($ )}

In this section, we assume that $R$ is regular of dimension $d \geqslant 1$ and mixed characteristic $(0, p)$ and study condition $(\natural)$ introduced in Theorem 1.1. Let $C(k)$ be a Cohen ring with residue field $k$ which is a coefficient ring of $\widehat{R}$, as in the Cohen structure theorem for complete local noetherian rings of mixed characteristic (see [GD64, Chapitre 0, Section 19.8] or [Mat89, Section 29]). Thus $C(k)$ is a subring of $\widehat{R}$. Let $y_{1}, \ldots, y_{d}$ be a regular system of parameters of $\widehat{R}$. The natural $C(k)$-algebra homomorphism $\varrho: C(k)\left[\left[x_{1}, \ldots, x_{d}\right]\right] \rightarrow \widehat{R}$ that maps $x_{i}$ to $y_{i}$ is onto. This implies that we can identify

$$
\widehat{R}=C(k)\left[\left[x_{1}, \ldots, x_{d}\right]\right] /(h)
$$

for some element $h \in \operatorname{Ker}(\varrho) \subset C(k)\left[\left[x_{1}, \ldots, x_{d}\right]\right]$ whose reduction $\bar{h}$ modulo $p$ is a non-zero element of the ideal $\left(x_{1}, \ldots, x_{d}\right)$ of $k\left[\left[x_{1}, \ldots, x_{d}\right]\right]$. The reduction $h_{p}$ of $h$ modulo the ideal $\left(x_{1}, \ldots, x_{d}\right)$ 


\section{Purity For BARsotti-TATE Groups}

of $C(k)\left[\left[x_{1}, \ldots, x_{d}\right]\right]$ is an element of $C(k)$ with the property that $C(k) /\left(h_{p}\right)=\widehat{R} /\left(y_{1}, \ldots, y_{d}\right)=k$. Thus $\left(h_{p}\right)=(p) \subset C(k)$, and therefore $h_{p}$ is $p$ times a unit of $C(k)$.

The ring $R / p R$ is regular if and only if $\bar{h} \notin\left(x_{1}, \ldots, x_{d}\right)^{2}$ and if and only if $\widehat{R}$ is isomorphic to $C(k)\left[\left[x_{1}, \ldots, x_{d-1}\right]\right]$. If $R / p R$ is regular and $\theta: C(k)\left[\left[x_{1}, \ldots, x_{d}\right]\right] \rightarrow \widehat{R}$ is a $C(k)$-algebra surjection, the images of $x_{1}, \ldots, x_{d}$ in $\widehat{R}$ do not necessarily form a regular system of parameters, but in all cases $\operatorname{Ker}(\theta)$ is generated by an element of $C(k)\left[\left[x_{1}, \ldots, x_{d}\right]\right]$ whose reduction modulo $p$ belongs to a regular system of parameters of $k\left[\left[x_{1}, \ldots, x_{d}\right]\right]$ and thus differs from $\bar{h}$ by a $k$-algebra automorphism of $k\left[\left[x_{1}, \ldots, x_{d}\right]\right]$. If $R / p R$ is not regular, then there exists no $C(k)$-algebra surjection $\theta: C(k)\left[\left[x_{1}, \ldots, x_{d}\right]\right] \rightarrow \widehat{R}$ under which the images of $x_{1}, \ldots, x_{d}$ in $\widehat{R}$ do not form a regular system of parameters of $\widehat{R}$.

If $C^{\prime}(k)$ is another Cohen ring which is a coefficient ring of $\widehat{R}$ and if $\varrho^{\prime}: C^{\prime}(k)\left[\left[x_{1}, \ldots, x_{d}\right]\right] \rightarrow$ $\widehat{R}$ is a $C^{\prime}(k)$-algebra surjection such that the images of $x_{1}, \ldots, x_{d}$ form a regular system of parameters of $\widehat{R}$, then there exists an isomorphism $\nu: C(k)\left[\left[x_{1}, \ldots, x_{d}\right]\right] \rightarrow C^{\prime}(k)\left[\left[x_{1}, \ldots, x_{d}\right]\right]$ such that we have $\varrho^{\prime}=\varrho \circ \nu$. To check this, observe that up to a $C^{\prime}(k)$-algebra automorphism of $C^{\prime}(k)\left[\left[x_{1}, \ldots, x_{d}\right]\right]$ preserving the ideal $\left(x_{1}, \ldots, x_{d}\right)$, we can assume that $\varrho^{\prime}\left(x_{i}\right)=y_{i}$ for all $i \in$ $\{1, \ldots, d\}$. As the homomorphism $\mathbb{Z}_{p} \rightarrow C(k)$ is formally smooth, there exists a homomorphism $\nu_{p}: C(k) \rightarrow C^{\prime}(k)\left[\left[x_{1}, \ldots, x_{d}\right]\right]$ whose composite with $\varrho^{\prime}$ is the inclusion $C(k) \rightarrow \widehat{R}$. Thus we can take $\nu$ such that it extends $\nu_{p}$ and maps $x_{i}$ into $x_{i}$ for all $i \in\{1, \ldots, d\}$.

From the last two paragraphs, we get that the ideal $(\bar{h})$ of $k\left[\left[x_{1}, \ldots, x_{d}\right]\right]$ coming from a presentation of $\widehat{R}$ as $C(k)\left[\left[x_{1}, \ldots, x_{d}\right]\right] /(h)$ is uniquely determined by $R$ up to automorphisms of $k\left[\left[x_{1}, \ldots, x_{d}\right]\right]$ inducing the identity on the residue field $k$, and $\bar{h}$ is uniquely determined up to units and such automorphisms. We say that $\bar{h}$ is a Cohen element of $R$. Thus condition ( $($ ) for $R$ means that one Cohen element of $R$ does not belong to the ideal $\left(x_{1}^{p}, \ldots, x_{d}^{p}\right)+\left(x_{1}, \ldots, x_{d}\right)^{2 p-2}$ of $k\left[\left[x_{1}, \ldots, x_{d}\right]\right]$; equivalently, all Cohen elements of $R$ have this property.

Recall [Mat89, Exercise 14.5] that the multiplicity of $R / p R$ is the order of vanishing of $p$; that is, it is the positive integer $e$ such that $p \in \mathfrak{m}^{e} \backslash \mathfrak{m}^{e+1}$, where $\mathfrak{m}$ is the maximal ideal of $R$. As $\widehat{R} / p \widehat{R} \simeq k\left[\left[x_{1}, \ldots, x_{d}\right]\right] /(\bar{h})$, the integer $e$ is also the order of vanishing of $\bar{h}$. In particular,

(i) if $e<p$, then condition ( $($ ) holds;

(ii) if condition ( $($ ) holds, then $e \leqslant 2 p-3$ for $d \geqslant 2$ and $e \leqslant p-1$ for $d=1$.

FACT 3.1. Suppose that $\widehat{R}$ is presented as $C(k)\left[\left[x_{1}, \ldots, x_{d}\right]\right] /(h)$. Let $l$ be a field extension of $k$, and let $C(k) \rightarrow C(l)$ be a monomorphism between Cohen rings which modulo $p$ is the inclusion $k \rightarrow l$. Then the $R$-algebra $R^{\prime}:=C(l)\left[\left[x_{1}, \ldots, x_{d}\right]\right] /(h)$ is faithfully flat, $R^{\prime}$ is regular, and condition ( $\left(\right.$ ) holds for $R$ if and only if it holds for $R^{\prime}$.

Proof. As the $C(k)$-algebra $C(l)$ is faithfully flat, the $C(k)\left[x_{1}, \ldots, x_{d}\right]$-algebra $C(l)\left[x_{1}, \ldots, x_{d}\right]$ is also faithfully flat. Taking completions of localizations at maximal ideals $\left(p, x_{1}, \ldots, x_{d}\right)$, we get that the $C(k)\left[\left[x_{1}, \ldots, x_{d}\right]\right]$-algebra $C(l)\left[\left[x_{1}, \ldots, x_{d}\right]\right]$ is flat; see [Mat89, Theorem 22.4(i)]. Hence $\widehat{R} \rightarrow R^{\prime}$ is flat; being local, it is faithfully flat. The same holds for $R \rightarrow R^{\prime}$, and $R^{\prime}$ is regular by [Mat89, Theorem 23.7]. The last assertion is clear.

\subsection{Taking slices}

In this subsection, we study for $d \geqslant 3$ the behavior of condition ( $\downarrow$ ) under restrictions to quotient rings of $\widehat{R}$ which are regular of dimension $d-1$. We have the following general and abstract lemma on homogeneous polynomials which also holds for $d=2$. 


\section{O. GabBer And A. VAsiu}

Lemma 3.2. Let $d \geqslant 2$ be an integer. For $c \in\{1, \ldots,(d-1)(p-1)\}$, let $\mathbb{I}_{c}$ be the subset of $\{0, \ldots, p-1\}^{d}$ formed by all those $d$-tuples whose entries sum up to $c$. Let $l$ be a field of characteristic $p$. We consider a non-zero homogeneous polynomial

$$
\bar{h}_{c}\left(x_{1}, \ldots, x_{d}\right)=\sum_{\left(i_{1}, \ldots, i_{d}\right) \in \mathbb{I}_{c}} \delta_{i_{1}, \ldots, i_{d}} \prod_{t=1}^{d} x_{t}^{i_{t}} \in l\left[x_{1}, \ldots, x_{d}\right]
$$

of degree $c$. Then there exists an open dense subscheme $\mathbb{O}$ of the affine scheme $\mathbb{A}_{l}^{d-1}$ such that for each $(d-1)$-tuple $\left(v_{1}, \ldots, v_{d-1}\right) \in \mathbb{O}(l) \subset \mathbb{A}_{l}^{d-1}(l)=l^{d-1}$, the homogeneous polynomial

$$
\bar{q}_{c}\left(x_{1}, \ldots, x_{d-1}\right):=\bar{h}_{c}\left(x_{1}, \ldots, x_{d-1}, \sum_{t=1}^{d-1} v_{t} x_{t}\right)
$$

has a non-zero image in $l\left[x_{1}, \ldots, x_{d-1}\right] /\left(x_{1}^{p}, \ldots, x_{d-1}^{p}\right)$.

Proof. For $q \in \mathbb{N} \cap\{c-p+1, \ldots, c\}$, let $\mathbb{J}_{q}^{d-1}$ be the subset of $\{0, \ldots, p-1\}^{d-1}$ formed by all those $(d-1)$-tuples whose entries sum up to $q$. For $\left(j_{1}, \ldots, j_{d-1}\right) \in \mathbb{J}_{c}^{d-1}$, the coefficient of $\prod_{t=1}^{d-1} x_{t}^{j_{t}}$ in $\bar{q}_{c}$ is a sum of the form

$$
\sum_{q \in \mathbb{N} \cap\{c-p+1, \ldots, c\}} \sum_{\left(i_{1}, \ldots, i_{d-1}\right) \in \mathbb{J}_{q}^{d-1}, i_{1} \leqslant j_{1}, \ldots, i_{d-1} \leqslant j_{d-1}} \gamma_{i_{1}, \ldots, i_{d-1}}^{j_{1}, \ldots, j_{d-1}} \delta_{i_{1}, \ldots, i_{d-1}, c-q} \prod_{t=1}^{d-1} v_{t}^{j_{t}-i_{t}}
$$

where each $\gamma_{i_{1}, \ldots, i_{d-1}}^{j_{1}, \ldots, j_{d-1}}$ is a multinomial coefficient that divides $(c-q)$ ! and thus is a non-zero element of $l$.

If $\bar{h}_{c}$ is a polynomial only in the indeterminates $x_{1}, \ldots, x_{d-1}$, then $\bar{q}_{c}=\bar{h}_{c}$ does not depend on the $(d-1)$-tuple $\left(v_{1}, \ldots, v_{d-1}\right)$ and has a non-zero image in $l\left[x_{1}, \ldots, x_{d-1}\right] /\left(x_{1}^{p}, \ldots, x_{d-1}^{p}\right)$; thus in this case we can take $\mathbb{O}:=\mathbb{A}_{l}^{d-1}$.

Therefore, we can assume that $\bar{h}_{c}$ is not a polynomial only in the indeterminates $x_{1}, \ldots, x_{d-1}$; that is, there exists a non-zero coefficient $\delta_{i_{1}, \ldots, i_{d}}$ of $\bar{h}_{c}$ with $i_{d} \geqslant 1$ or, equivalently, with $\sum_{t=1}^{d-1} i_{t}<c$. As $c \leqslant(d-1)(p-1)$, if for each $t \in\{1, \ldots, d-1\}$, we consider an element $j_{t} \in\left\{i_{t}, \ldots, p-1\right\}$ such that we have $\sum_{t=1}^{d-1} j_{t}=c$, then the coefficient of $\prod_{t=1}^{d-1} x_{t}^{j_{t}}$ in $\bar{q}_{c}$ is a non-zero polynomial $\bar{q}_{c, j_{1}, \ldots, j_{d-1}}\left(v_{1}, \ldots, v_{d-1}\right) \in l\left[v_{1}, \ldots, v_{d-1}\right]$. Let $\mathbb{O}$ be the principal open subscheme of $\mathbb{A}_{l}^{d-1}$ which is the complement of the zero locus of $\bar{q}_{c, j_{1}, \ldots, j_{d-1}}\left(x_{1}, \ldots, x_{d-1}\right)$. Thus, if $\left(v_{1}, \ldots, v_{d-1}\right) \in \mathbb{O}(l)$, the coefficient of $\prod_{t=1}^{d-1} x_{t}^{j_{t}}$ in $\bar{q}_{c}$ is non-zero, and therefore the image of $\bar{q}_{c}$ in $l\left[x_{1}, \ldots, x_{d-1}\right] /\left(x_{1}^{p}, \ldots, x_{d-1}^{p}\right)$ is non-zero.

Proposition 3.3. We assume that $d \geqslant 3$, that $k$ is an infinite field, that $R=\widehat{R}$ is complete and that condition $(\emptyset)$ holds for $R$. Then for each $d_{1} \in\{2, \ldots, d-1\}$, there exists a closed regular subscheme $\operatorname{Spec}(S)$ of $X=\operatorname{Spec}(R)$ of dimension $d_{1}$ and mixed characteristic $(0, p)$ such that condition ( $($ ) holds for $S$.

Proof. Using induction on $d \geqslant 3$, it suffices to consider the case where $d_{1}=d-1$. We write $\bar{h}=\bar{h}_{0}+\sum_{i=1}^{2 p-3} \bar{h}_{i}$, where $\bar{h}_{0}$ belongs to the ideal $\left(x_{1}^{p}, \ldots, x_{d}^{p}\right)+\left(x_{1}, \ldots, x_{d}\right)^{2 p-2}$ of $k\left[\left[x_{1}, \ldots, x_{d}\right]\right]$ and where for $i \in\{1, \ldots, 2 p-3\}$, the polynomial $\bar{h}_{i}$ is (possibly 0 ) homogeneous of degree $i$ in the indeterminates $x_{1}, \ldots, x_{d}$ and involves only monomials with exponents less than $p$. Let $c \in\{1, \ldots, 2 p-3\}$ be the smallest integer such that $\bar{h}_{c}$ is non-zero (it exists as condition ( $\bullet$ ) holds for $R$ ). The field $k$ is infinite, and we have $c \leqslant 2 p-3<(d-1)(p-1)$ as $d \geqslant 3$. Thus from Lemma 3.2 applied with $l=k$, we get that there exists a $(d-1)$-tuple $\left(v_{1}, \ldots, v_{d-1}\right) \in \mathbb{O}(k) \subset k^{d-1}$ such 


\section{PURITy FOR BARsotTi-TATE Groups}

that the homogeneous polynomial $\bar{h}_{c}\left(x_{1}, \ldots, x_{d-1}, \sum_{t=1}^{d-1} v_{t} x_{t}\right)$ of degree $c$ has a non-zero image in $k\left[\left[x_{1}, \ldots, x_{d-1}\right]\right] /\left(x_{1}^{p}, \ldots, x_{d-1}^{p}\right)$. This implies that $\bar{h}\left(x_{1}, \ldots, x_{d-1}, \sum_{t=1}^{d-1} v_{t} x_{t}\right)$ does not belong to the ideal $\left(x_{1}^{p}, \ldots, x_{d-1}^{p}\right)+\left(x_{1}, \ldots, x_{d-1}\right)^{2 p-2}$ of $k\left[\left[x_{1}, \ldots, x_{d-1}\right]\right]$, and therefore condition ( $($ ) holds for the regular local ring

$$
S:=R /\left(x_{d}-\sum_{t=1}^{d-1} w_{i} x_{i}\right)=C(k)\left[\left[x_{1}, \ldots, x_{d-1}\right]\right] /\left(h\left(x_{1}, \ldots, x_{d-1}, \sum_{t=1}^{d-1} w_{t} x_{t}\right)\right)
$$

of dimension $d-1$ and mixed characteristic $(0, p)$, where $\left(w_{1}, \ldots, w_{d-1}\right) \in C(k)^{d-1}$ is such that its reduction modulo $p$ is $\left(v_{1}, \ldots, v_{d-1}\right) \in k^{d-1}$.

\subsection{Functorial properties}

In this subsection, we study the behavior of condition ( $q$ ) under local homomorphisms. We consider a local homomorphism $\varphi: R \rightarrow R^{\prime}$ between regular local rings of mixed characteristic $(0, p)$. Let $d^{\prime} \geqslant 1$ be the dimension of $R^{\prime}$. Let $k^{\prime}$ be the residue field of $R^{\prime}$, and let $C\left(k^{\prime}\right)$ be a Cohen ring which is a coefficient ring of $\widehat{R^{\prime}}$. Let $y_{1}^{\prime}, \ldots, y_{d^{\prime}}^{\prime}$ be a regular system of parameters of $\widehat{R^{\prime}}$. The natural $C\left(k^{\prime}\right)$-algebra homomorphism $\varrho^{\prime}: C\left(k^{\prime}\right)\left[\left[x_{1}^{\prime}, \ldots, x_{d^{\prime}}^{\prime}\right]\right] \rightarrow \widehat{R^{\prime}}$ that maps $x_{i}^{\prime}$ to $y_{i}^{\prime}$ is onto, and we identify

$$
\widehat{R^{\prime}}=C\left(k^{\prime}\right)\left[\left[x_{1}^{\prime}, \ldots, x_{d^{\prime}}^{\prime}\right]\right] /\left(h^{\prime}\right)
$$

for some element $h^{\prime} \in C\left(k^{\prime}\right)\left[\left[x_{1}^{\prime}, \ldots, x_{d^{\prime}}^{\prime}\right]\right]$. As the homomorphism $\mathbb{Z}_{p} \rightarrow C(k)$ is formally smooth, the composite homomorphism $C(k) \rightarrow \widehat{R} \rightarrow \widehat{R^{\prime}}$ admits a lift to a homomorphism

$$
\Phi_{p}: C(k) \rightarrow C\left(k^{\prime}\right)\left[\left[x_{1}^{\prime}, \ldots, x_{d^{\prime}}^{\prime}\right]\right] .
$$

We consider a homomorphism $\Phi: C(k)\left[\left[x_{1}, \ldots, x_{d}\right]\right] \rightarrow C\left(k^{\prime}\right)\left[\left[x_{1}^{\prime}, \ldots, x_{d^{\prime}}\right]\right]$ that extends $\Phi_{p}$, that maps each $x_{i}$ into the ideal $\left(x_{1}^{\prime}, \ldots, x_{d^{\prime}}^{\prime}\right)$ of $C\left(k^{\prime}\right)\left[\left[x_{1}^{\prime}, \ldots, x_{d^{\prime}}\right]\right]$ and that is such that we have a commutative diagram

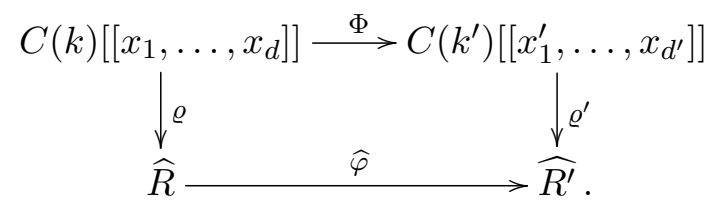

Proposition 3.4. With $\varphi$ and $\Phi$ as above, the following three properties hold:

(a) We have an equality $(\Phi(h))=\left(h^{\prime}\right)$ of ideals of $C\left(k^{\prime}\right)\left[\left[x_{1}^{\prime}, \ldots, x_{d^{\prime}}^{\prime}\right]\right.$.

(b) If condition ( $\left(\right.$ ) holds for $R^{\prime}$, then it also holds for $R$.

(c) Let $X^{\prime}:=\operatorname{Spec}\left(R^{\prime}\right)$. We assume that the morphism $X^{\prime} \rightarrow X$ defined by $\varphi$ is flat and its fiber over the closed point of $X$ is regular (that is, the local ring $R^{\prime} /\left(\varphi\left(y_{1}\right), \ldots, \varphi\left(y_{d}\right)\right) R^{\prime}$ is regular). Then condition ( $\bullet$ ) holds for $R$ if and only if it holds for $R^{\prime}$.

Proof. As $\Phi$ induces $\widehat{\varphi}: \widehat{R} \rightarrow \widehat{R^{\prime}}$, we have $\Phi(h) \in\left(h^{\prime}\right)$. We consider the unique element $u$ of $C\left(k^{\prime}\right)\left[\left[x_{1}^{\prime}, \ldots, x_{d^{\prime}}^{\prime}\right]\right]$ such that we have $\Phi(h)=h^{\prime} u$. To prove statement (a), it suffices to show that $u$ is a unit of $C\left(k^{\prime}\right)\left[\left[x_{1}^{\prime}, \ldots, x_{d^{\prime}}^{\prime}\right]\right]$. If $h_{p}^{\prime}$ and $u_{p}$ are the reductions modulo the ideal $\left(x_{1}^{\prime}, \ldots, x_{d^{\prime}}^{\prime}\right)$ of $C\left(k^{\prime}\right)\left[\left[x_{1}^{\prime}, \ldots, x_{d^{\prime}}^{\prime}\right]\right]$, then the equality $\Phi(h)=h^{\prime} u$ modulo the ideal $\left(x_{1}^{\prime}, \ldots, x_{d^{\prime}}^{\prime}\right)$ becomes an identity $\varphi_{p}\left(h_{p}\right)=h_{p}^{\prime} u_{p}$ between elements of $C\left(k^{\prime}\right)$, where

$$
\varphi_{p}: C(k) \rightarrow C\left(k^{\prime}\right)=C\left(k^{\prime}\right)\left[\left[x_{1}^{\prime}, \ldots, x_{d^{\prime}}^{\prime}\right]\right] /\left(x_{1}^{\prime}, \ldots, x_{d^{\prime}}^{\prime}\right)
$$

is induced by $\Phi_{p}$. We know that $h_{p}$ and $h_{p}^{\prime}$ are $p$ times units of $C(k)$ and $C\left(k^{\prime}\right)$, respectively; see 


\section{O. Gabber and A. Vasiu}

the beginning of Section 3. From the last two sentences, we get that $u_{p}$ is a unit of $C\left(k^{\prime}\right)$ and therefore $u$ is a unit of $C\left(k^{\prime}\right)\left[\left[x_{1}^{\prime}, \ldots, x_{d^{\prime}}^{\prime}\right]\right]$. Thus, statement (a) holds.

To prove statement (b), let $\bar{h}^{\prime}$ and $\bar{\Phi}: k\left[\left[x_{1}, \ldots, x_{d}\right] \rightarrow k^{\prime}\left[\left[x_{1}^{\prime}, \ldots, x_{d^{\prime}}^{\prime}\right]\right]\right.$ be the reductions modulo $p$ of $h^{\prime}$ and $\Phi$, respectively. The elements $\bar{\Phi}(\bar{h})$ and $\bar{h}^{\prime}$ differ by a unit by statement (a). Thus, if $\bar{h}$ belongs to the ideal $\left(x_{1}^{p}, \ldots, x_{d}^{p}\right)+\left(x_{1}, \ldots, x_{d}\right)^{2 p-2}$ of $k\left[\left[x_{1}, \ldots, x_{d}\right]\right]$, then $\bar{\Phi}(\bar{h})$ and $\bar{h}^{\prime}$ belong to the ideal of $k^{\prime}\left[\left[x_{1}^{\prime}, \ldots, x_{d^{\prime}}^{\prime}\right]\right]$ generated by $\bar{\Phi}\left(\left(x_{1}^{p}, \ldots, x_{d}^{p}\right)\right)+\bar{\Phi}\left(\left(x_{1}, \ldots, x_{d}\right)^{2 p-2}\right)$ and thus to the ideal $\left(\left(x_{1}^{\prime}\right)^{p}, \ldots,\left(x_{d^{\prime}}^{\prime}\right)^{p}\right)+\left(x_{1}^{\prime}, \ldots, x_{d^{\prime}}^{\prime}\right)^{2 p-2}$ of $k^{\prime}\left[\left[x_{1}^{\prime}, \ldots, x_{d}^{\prime}\right]\right]$, and therefore condition ( $\bullet$ ) does not hold for $R^{\prime}$. From this, statement (b) follows.

Based on statement (b), to prove statement (c) it suffices to show that if condition ( $\square$ ) holds for $R$, then it also holds for $R^{\prime}$. To ease notation, we can assume that $R$ and $R^{\prime}$ are complete; thus $\varphi=\widehat{\varphi}, y_{1}, \ldots, y_{d} \in R$ and $y_{1}^{\prime}, \ldots, y_{d^{\prime}}^{\prime} \in R^{\prime}$. Let $c$ be the dimension of the regular local ring

$$
\bar{R}^{\prime}:=R^{\prime} /\left(\varphi\left(y_{1}\right), \ldots, \varphi\left(y_{d}\right)\right) R^{\prime}
$$

of characteristic $p$. As $\varphi$ is flat, we have $d^{\prime}=d+c$; see [Mat89, Theorem 15.1]. We can assume that $y_{d+1}^{\prime}, \ldots, y_{d+c}^{\prime}$ map into a regular system of parameters of $\bar{R}^{\prime}$. Let $R_{1}^{\prime}=R^{\prime} /\left(y_{d+1}^{\prime}, \ldots, y_{d+c}^{\prime}\right)$; it is a regular local ring of dimension $d=d^{\prime}-c$. The composite homomorphism $\varphi_{1}: R \rightarrow R_{1}^{\prime}$ between local rings of dimension $d$ is such that $R_{1}^{\prime} /\left(\varphi\left(y_{1}\right), \ldots, \varphi\left(y_{d}\right)\right)=\operatorname{Spec}\left(k^{\prime}\right)$. This implies that $\varphi_{1}$ is flat; see [Mat89, Theorem 23.1]. Thus $R_{1}^{\prime}$ has mixed characteristic $(0, p)$. If condition ( $($ ) holds for $R_{1}^{\prime}$, then it also holds for $R^{\prime}$ by statement (b), and therefore by replacing $R^{\prime}$ with $R_{1}^{\prime}$, we can assume that $d^{\prime}=d$ and $c=0$, and therefore that we have $y_{i}^{\prime}=\varphi\left(y_{i}\right)$ for all $i \in\{1, \ldots, d\}$. We can also assume that we have $x_{i}^{\prime}=\Phi\left(x_{i}\right)$ for all $i \in\{1, \ldots, d\}$, that $\bar{\Phi}: k\left[\left[x_{1}, \ldots, x_{d}\right] \rightarrow k^{\prime}\left[\left[x_{1}^{\prime}, \ldots, x_{d^{\prime}}^{\prime}\right]\right]\right.$ can be identified with the canonical inclusion $k\left[\left[x_{1}, \ldots, x_{d}\right] \rightarrow k^{\prime}\left[\left[x_{1}, \ldots, x_{d}\right]\right]\right.$ and that under this identification, we have $\bar{h}=\bar{h}^{\prime}$. As $\bar{h}$ does not belong to the ideal $\left(x_{1}^{p}, \ldots, x_{d}^{p}\right)+\left(x_{1}, \ldots, x_{d}\right)^{2 p-2}$ of $k\left[\left[x_{1}, \ldots, x_{d}\right]\right]$, it does not belong to the ideal $\left(x_{1}^{p}, \ldots, x_{d}^{p}\right)+\left(x_{1}, \ldots, x_{d}\right)^{2 p-2}$ of $k^{\prime}\left[\left[x_{1}, \ldots, x_{d}\right]\right]$, and thus condition ( $\left(\right.$ ) holds for $R^{\prime}$. Thus, statement (c) holds.

\subsection{A generization property}

Proposition 3.5. We assume that $R$ is a regular local ring of dimension $d \geqslant 2$ and mixed characteristic $(0, p)$ for which condition $(\emptyset)$ holds. Let $R_{1}$ be a local ring of $X=\operatorname{Spec}(R)$ which is of mixed characteristic $(0, p)$ and dimension $d_{1} \in\{1, \ldots, d-1\}$. Then condition ( $($ ) holds for $R_{1}$.

Proof. Let $k_{1}$ be the residue field of $R_{1}$. Let $\mathfrak{p}_{1}$ be the prime ideal of $R$ such that $R_{1}=R_{\mathfrak{p}_{1}}$. We consider two cases, as follows.

Case 1. We first consider the case where $R=\widehat{R}$ is complete. We fix an identification

$$
R=C(k)\left[\left[x_{1}, \ldots, x_{d}\right]\right] /(h),
$$

where $h \in C(k)\left[\left[x_{1}, \ldots, x_{d}\right]\right]$ is such that its reduction $\bar{h}$ modulo $p$ does not belong to the ideal $\left(x_{1}^{p}, \ldots, x_{d}^{p}\right)+\left(x_{1}, \ldots, x_{d}\right)^{2 p-2}$ of $k\left[\left[x_{1}, \ldots, x_{d}\right]\right]$. Let

$$
\mathfrak{p}:=\varrho^{-1}\left(\mathfrak{p}_{1}\right) \in \operatorname{Spec}\left(C(k)\left[\left[x_{1}, \ldots, x_{d}\right]\right]\right),
$$

let $\mathfrak{n}:=\mathfrak{p} C(k)\left[\left[x_{1}, \ldots, x_{d}\right]\right]_{\mathfrak{p}}$ be the maximal ideal of $C(k)\left[\left[x_{1}, \ldots, x_{d}\right]\right]_{\mathfrak{p}}$, and let $\mathfrak{n}^{(p)}$ be the $\mathfrak{n}$ primary ideal of $C(k)\left[\left[x_{1}, \ldots, x_{d}\right]\right]_{\mathfrak{p}}$ generated by $p$ and the $p$ th powers of elements of $\mathfrak{n}$. Let $\mathfrak{A}:=C(k)\left[\left[x_{1}, \ldots, x_{d}\right]\right]_{\mathfrak{p}} / \mathfrak{n}^{(p)} ;$ it is a local artinian ring which contains $k$, which has $\mathfrak{j}:=\mathfrak{n} / \mathfrak{n}^{(p)}$ as its maximal ideal and which as a $k$-algebra is isomorphic to $k_{1}\left[y_{1}, \ldots, y_{d_{1}}\right] /\left(y_{1}^{p}, \ldots, y_{d_{1}}^{p}\right)$.

As $\bar{h}$ does not belong to the ideal $\left(x_{1}^{p}, \ldots, x_{d}^{p}\right)+\left(x_{1}, \ldots, x_{d}\right)^{2 p-2}$ of the ring $k\left[\left[x_{1}, \ldots, x_{d}\right]\right]$, 


\section{PURITy FOR BARsotTi-TATE Groups}

we can speak about the smallest integer $n \in\{1, \ldots, 2 p-3\}$ such that $\bar{h}$ has in its writing a non-zero term of the form $v \prod_{j=1}^{d} x_{j}^{i_{j}}$ with each $i_{j} \in\{0, \ldots, p-1\}$ such that $n=\sum_{j=1}^{d} i_{j}$ and with $v \in k \backslash\{0\}$. This implies that there exist $n$ derivations $\partial_{1}, \ldots, \partial_{n}$ of $C(k)\left[\left[x_{1}, \ldots, x_{d}\right]\right]$ which are $C(k)$-linear, which belong to the set $\left\{\partial / \partial x_{1}, \ldots, \partial / \partial x_{d}\right\}$ and for which the element $\left(\partial_{1} \circ \ldots \circ \partial_{n}\right)(h)$ is a unit of $C(k)\left[\left[x_{1}, \ldots, x_{d}\right]\right]$ (for example, we can take $\partial_{1}, \ldots, \partial_{i_{1}}$ to be $\partial / \partial x_{1}$, $\partial_{i_{1}+1}, \ldots, \partial_{i_{1}+i_{2}}$ to be $\partial / \partial x_{2}, \ldots, \partial_{i_{1}+\cdots+i_{d-1}+1}, \ldots, \partial_{n}$ to be $\left.\partial / \partial x_{d}\right)$. For $i \in\{1, \ldots, n\}$, the derivation of $C(k)\left[\left[x_{1}, \ldots, x_{d}\right]\right]_{\mathfrak{p}}$ that extends $\partial_{i}$ induces a derivation $\bar{\partial}_{i}: \mathfrak{A} \rightarrow \mathfrak{A}$.

Let $\bar{h}_{(p)}$ be the image of $\bar{h}$ in $\mathfrak{A}$. As $\left(\partial_{1} \circ \cdots \circ \partial_{n}\right)(h)$ is a unit of $C(k)\left[\left[x_{1}, \ldots, x_{d}\right]\right]$, the element $\left(\bar{\partial}_{1} \circ \cdots \circ \bar{\partial}_{n}\right)\left(\bar{h}_{(p)}\right)$ is a unit of $\mathfrak{A}$. We show that the assumption that $\bar{h}_{(p)} \in \mathfrak{j}^{2 p-2}$ leads to a contradiction. By a decreasing induction on $i \in\{1, \ldots, n\}$, we get that $\left(\bar{\partial}_{i} \circ \cdots \circ \bar{\partial}_{n}\right)\left(\bar{h}_{(p)}\right)$ belongs to $\mathrm{j}^{2 p-2-n+i-1}$. Thus the element $\left(\bar{\partial}_{1} \circ \cdots \circ \bar{\partial}_{n}\right)\left(\bar{h}_{(p)}\right)$ belongs to $\mathfrak{j}^{2 p-2-n}$. From this and the inequality $2 p-2-n \geqslant 1$, we get that $\left(\bar{\partial}_{1} \circ \cdots \circ \bar{\partial}_{n}\right)\left(\bar{h}_{(p)}\right) \in \mathfrak{j}$ is not a unit of $\mathfrak{A}$. This gives a contradiction. Thus $\bar{h}_{(p)} \notin \mathfrak{j}^{2 p-2}$. This implies that $h$ does not belong to the ideal $\mathfrak{n}^{(p)}+\mathfrak{n}^{2 p-2}$ of $C(k)\left[\left[x_{1}, \ldots, x_{d}\right]\right]_{\mathfrak{p}}$.

Let $C\left(k_{1}\right)$ be a Cohen ring which is a coefficient ring of the completion $\overline{C(k)\left[\left[x_{1}, \ldots, x_{d}\right]\right]_{\mathfrak{p}}}$. We fix a $C\left(k_{1}\right)$-algebra isomorphism

$$
\overline{C(k)\left[\left[x_{1}, \ldots, x_{d}\right]\right]_{\mathfrak{p}}} \simeq C\left(k_{1}\right)\left[\left[y_{1}, \ldots, y_{d_{1}}\right]\right]
$$

which lifts the $k_{1}$-algebra isomorphism $\mathfrak{A} / \mathfrak{j} \simeq k_{1}\left[y_{1}, \ldots, y_{d_{1}}\right] /\left(y_{1}^{p}, \ldots, y_{d_{1}}^{p}\right)$. The completion $\widehat{R_{1}}$ of $R_{1}$ is canonically identified with $\overline{C(k)\left[\left[x_{1}, \ldots, x_{d}\right]_{\mathfrak{p}}\right.} /(h)$ and thus with $C\left(k_{1}\right)\left[\left[y_{1}, \ldots, y_{d_{1}}\right]\right] /(h)$. From the last sentence of the previous paragraph, we get that the image of $\bar{h}$ in $\left(y_{1}, \ldots, y_{d_{1}}\right)$ does not belong to the ideal $\left(y_{1}^{p}, \ldots, y_{d_{1}}^{p}\right)+\left(y_{1}, \ldots, y_{d_{1}}\right)^{2 p-2}$ of $k_{1}\left[\left[y_{1}, \ldots, y_{d_{1}}\right]\right]$. Thus condition ( $($ ) holds for $R_{1}$ if $R=\widehat{R}$ is complete.

Case 2. We consider the case where $R$ is not complete. Let $\mathfrak{q}$ be a prime ideal of $\widehat{R}$ which is minimal over $\mathfrak{p}_{1} \widehat{R}$. We have a canonical homomorphism $R_{1}=R_{\mathfrak{p}_{1}} \rightarrow \widehat{R}_{\mathfrak{q}}$ of local rings. From case 1 applied to $\widehat{R}$, we get that condition ( $\natural$ ) holds for $\widehat{R}_{\mathfrak{q}}$. Thus condition ( $\natural$ ) also holds for $R_{1}$, by Proposition 3.4(b).

\section{On closed subschemes of projective spaces}

Let $l$ be a field. Let $N \in \mathbb{N}^{*}$. Let $\mathcal{C}$ be a closed connected (thus non-empty) subscheme of the projective space $\mathbb{P}_{l}^{N}$. Let $\widehat{\mathbb{P}_{l}^{N}}$ be the formal completion of $\mathbb{P}_{l}^{N}$ along $\mathcal{C}$. Let $\kappa$ be the field of rational functions of $\mathbb{P}_{l}^{N}$, and let $\kappa^{\prime}$ be the ring of formal-rational functions on $\widehat{\mathbb{P}_{l}^{N}}$. We recall from [HM68, Definition 2.9.3] that $\mathcal{C}$ is said to be G3 in $\mathbb{P}_{l}^{N}$ if the homomorphism $\kappa \rightarrow \kappa^{\prime}$ associated to the natural morphism $\chi: \widehat{\mathbb{P}_{l}^{N}} \rightarrow \mathbb{P}_{l}^{N}$ of locally ringed spaces is an isomorphism. For instance, from the proof of [HM68, Theorem 3.3], we get that if $l$ is an algebraically closed field and if $\mathcal{C}$ is irreducible of positive dimension, then $\mathcal{C}$ is (universally) G3 in $\mathbb{P}_{l}^{N}$. In Section 5, we will need the following general result of formal algebraic geometry.

LEMma 4.1. We assume that $\mathcal{C}$ is a geometrically connected scheme of positive dimension which is $G 3$ in $\mathbb{P}_{l}^{N}$. Let $\mathcal{U}$ be an open subscheme of $\mathbb{P}_{l}^{N}$ which contains $\mathcal{C}$. Let $\mathcal{V}$ be a torsion-free coherent sheaf on $\mathcal{U}$. Let $\widehat{\mathcal{V}}:=\chi^{*}(\mathcal{V})$ be the coherent sheaf on $\widehat{\mathbb{P}_{l}^{N}}$ which is the natural pullback of $\mathcal{V}$. Then there exists an open subscheme $\mathcal{W}$ of $\mathcal{U}$ which contains $\mathcal{C}$ and is such that the natural 
pullback homomorphism

$$
\rho_{\mathcal{W}}: H^{0}(\mathcal{W}, \mathcal{V}) \rightarrow H^{0}\left(\widehat{\mathbb{P}_{l}^{N}}, \widehat{\mathcal{V}}\right)
$$

between global sections is an isomorphism between finite-dimensional l-vector spaces. Moreover, if $\mathcal{V}$ is a reflexive $\mathcal{O}_{\mathcal{U}}$-module, then we can take $\mathcal{W}=\mathcal{U}$.

Proof. Using a meromorphic basis of $\mathcal{V}$ and the assumption that $\kappa=\kappa^{\prime}$, one sees that the finitedimensional $\kappa$-vector space $V$ of meromorphic sections of $\mathcal{V}$ maps isomorphically to the space of meromorphic sections of $\widehat{\mathcal{V}}$. Thus, as $\mathcal{V}$ is torsion-free, each homomorphism $\rho_{\mathcal{W}}$ is injective and we have natural inclusions $H^{0}(\mathcal{U}, \mathcal{V}) \subset H^{0}\left(\widehat{\mathbb{P}_{l}^{N}}, \widehat{\mathcal{V}}\right) \subset V$.

We will first show that for each $\xi \in H^{0}\left(\widehat{\mathbb{P}_{l}^{N}}, \widehat{\mathcal{V}}\right) \subset V$, there exists an open subscheme $\mathcal{W}_{\xi}$ of $\mathcal{U}$ which contains $\mathcal{C}$ and is such that we have $\xi \in H^{0}\left(\mathcal{W}_{\xi}, \mathcal{V}\right)$.

Let $\mathcal{O}$ be a local ring of $\mathbb{P}_{l}^{N}$ at a closed point $z$ of $\mathcal{C}$; it is a subring of $\kappa$. Let $\widehat{\mathcal{O}}$ be the completion of $\mathcal{O}$, and let $\mathcal{M}$ be the $\mathcal{O}$-submodule of $V$ defined by the global sections of $\mathcal{V}$ over $\operatorname{Spec}(\mathcal{O})$; we can identify $V=\kappa \otimes_{\mathcal{O}} \mathcal{M}$.

As the homomorphism $\mathcal{O} \rightarrow \widehat{\mathcal{O}}$ is faithfully flat, it is well known that we have $\kappa \cap \widehat{\mathcal{O}}=\mathcal{O}$ (the intersection being taken inside the field of fractions of $\widehat{\mathcal{O}}$ ). We recall the argument for this. For $a \in \kappa \cap \widehat{\mathcal{O}}$, we have inclusions of $\mathcal{O}$-modules $\mathcal{O} \subset \mathcal{O}+\mathcal{O} a \subset \kappa$. Tensoring with $\widehat{\mathcal{O}}$ over $\mathcal{O}$, we get inclusions $\widehat{\mathcal{O}}=\widehat{\mathcal{O}}+\widehat{\mathcal{O}} a \subset \widehat{\mathcal{O}} \otimes_{\mathcal{O}} \kappa$. As the homomorphism $\mathcal{O} \rightarrow \widehat{\mathcal{O}}$ is faithfully flat, it follows that the inclusion $\mathcal{O} \subset \mathcal{O}+\mathcal{O} a$ is an identity as well, and therefore we have $a \in \mathcal{O}$; thus $\kappa \cap \widehat{\mathcal{O}}=\mathcal{O}$.

As $\mathcal{M}$ is a torsion-free $\mathcal{O}$-module, the same argument shows that we have

$$
\mathcal{M}=\mathcal{O} \otimes_{\mathcal{O}} \mathcal{M}=\left(\kappa \otimes_{\mathcal{O}} \mathcal{M}\right) \cap\left(\widehat{\mathcal{O}} \otimes_{\mathcal{O}} \mathcal{M}\right)=V \cap\left(\widehat{\mathcal{O}} \otimes_{\mathcal{O}} \mathcal{M}\right) .
$$

But as $\xi \in H^{0}\left(\widehat{\mathbb{P}_{l}^{N}}, \widehat{\mathcal{V}}\right) \subset V$, we also have $\xi \in V \cap\left(\widehat{\mathcal{O}} \otimes_{\mathcal{O}} \mathcal{M}\right)$, and thus we have $\xi \in \mathcal{M}$. As $z$ is an arbitrary closed point of $\mathcal{C}$, we get that there exists an open subscheme $\mathcal{W}_{\xi}$ of $\mathcal{U}$ which contains $\mathcal{C}$ and is such that we have $\xi \in H^{0}\left(\mathcal{W}_{\xi}, \mathcal{V}\right)$.

In this paragraph, we consider the case where $\mathcal{V}$ is a reflexive $\mathcal{O}_{\mathcal{U}}$-module. As $\mathcal{C}$ has positive dimension, the complement of $\mathcal{W}_{\xi}$ in $\mathbb{P}_{l}^{N}$ has codimension in $\mathbb{P}_{l}^{N}$ at least 2 (as it does not intersect $\mathcal{C}$ ). This implies that $\xi \in H^{0}\left(\mathcal{W}_{\xi}, \mathcal{V}\right)=H^{0}(\mathcal{U}, \mathcal{V})$; use Fact 2.1(c) for the equality part. Thus we can take $\mathcal{W}_{\xi}=\mathcal{U}$. This implies that $\rho_{\mathcal{U}}$ is surjective and therefore an isomorphism. It is well known that $H^{0}(\mathcal{U}, \mathcal{V})$ is a finite-dimensional $l$-vector space (this can be checked by considering a coherent

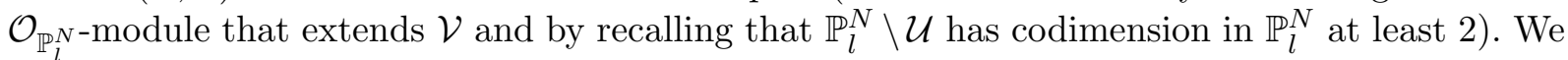
conclude that $H^{0}\left(\widehat{\mathbb{P}_{l}^{N}}, \widehat{\mathcal{V}}\right)$ is a finite-dimensional $l$-vector space when $\mathcal{V}$ is a reflexive $\mathcal{O}_{\mathcal{U}}$-module.

We now consider the general case where $\mathcal{V}$ is torsion-free but not necessarily a reflexive $\mathcal{O}_{\mathcal{U}}$ module. As $\mathcal{V}$ is torsion-free, it is a $\mathcal{O}_{\mathcal{U}}$-submodule of a coherent locally free $\mathcal{O}_{\mathcal{U}}$-module. From this and the previous paragraph, we get that $H^{0}\left(\widehat{\mathbb{P}_{l}^{N}}, \widehat{\mathcal{V}}\right)$ is a finite-dimensional $l$-vector space. Let $\xi_{1}, \ldots, \xi_{n} \in H^{0}\left(\widehat{\mathbb{P}_{l}^{N}}, \widehat{\mathcal{V}}\right) \subset V$ generate $H^{0}\left(\widehat{\mathbb{P}_{l}^{N}}, \widehat{\mathcal{V}}\right)$ as an $l$-vector space. For each $i \in\{1, \ldots, n\}$, let $\mathcal{W}_{\xi_{i}}$ be an open subscheme of $\mathcal{U}$ which contains $\mathcal{C}$ and is such that we have $\xi_{i} \in H^{0}\left(\mathcal{W}_{\xi_{i}}, \mathcal{V}\right)$ (see above). Then for the open subscheme $\mathcal{W}:=\cap_{i=1}^{n} \mathcal{W}_{i}$ of $\mathcal{U}$, the homomorphism $\rho_{\mathcal{W}}$ is surjective and therefore also an isomorphism between finite-dimensional $l$-vector spaces.

\section{Proof of Theorem 1.2}

In this section, we prove Theorem 1.2. By enlarging $l^{\prime}$ and making $\mathcal{C}$ smaller if needed, to prove Theorem 1.2, we can assume that $\mathcal{C}$ is a geometrically integral curve whose normalization $\mathcal{C}^{\text {n }}$ is 


\section{Purity For BARsotti-TATE GROUPS}

smooth over $\operatorname{Spec}\left(l^{\prime}\right)$. Let $\mathcal{I}$ be the ideal sheaf of $\mathcal{O}_{\mathbb{P}_{l^{\prime}}^{N}}$ which defines $\mathcal{C}$. We have the following general constancy result.

Proposition 5.1. In the context of Theorem 1.2, we assume that $l^{\prime}=l$ and that $\mathcal{C}$ is a geometrically integral curve with smooth normalization. Then the BT $\mathcal{D} \widehat{\mathbb{P}_{l}^{N}}$ over the formal completion $\widehat{\mathbb{P}_{l}^{N}}$ of $\mathbb{P}_{l}^{N}$ along $\mathcal{C}$ induced naturally by the inductive system $\mathcal{D}_{n, \mathcal{U}_{\infty}}$ is constant as well; that is, it is isomorphic to $G_{\widehat{\mathbb{P}_{l}^{N}}}$ with $G$ a BT over $\operatorname{Spec}(l)$.

Proof. We first consider the case where $\mathcal{C}=\mathcal{C}^{\text {n }}$ is smooth over $\operatorname{Spec}(l)$. The conormal sheaf $\mathcal{I} / \mathcal{I}^{2}$ of $\mathcal{C}$ inside $\mathbb{P}_{l}^{N}$ is a subsheaf of $\mathcal{O}_{\mathcal{C}} \otimes_{\mathcal{O}_{\mathbb{P}_{l}^{N}}} \Omega_{\mathbb{P}_{l}^{N}}^{1}$ and thus (by the Euler sequence) also of $\mathcal{O}_{\mathcal{C}}(-1)^{N+1}$. Thus for each $t \in \mathbb{N}^{*}$, its $t$ th symmetric power $S^{t}\left(\mathcal{I} / \mathcal{I}^{2}\right)=\mathcal{I}^{t} / \mathcal{I}^{t+1}$ has no non-zero global section.

We check by induction on $t \in \mathbb{N}$ that the BT given by the inductive system $\mathcal{D}_{n, \mathcal{C}_{t}}$ over the $t$ th infinitesimal neighborhood $\mathcal{C}_{t}$ of $\mathcal{C}$ in $\mathbb{P}_{l}^{N}$ is constant. We recall the convention of [GD67, Definition 16.1.2] that $\mathcal{C}_{t}$ is defined by the ideal $\mathcal{I}^{t+1}$ of $\mathbb{P}_{l}^{N}$. As the inductive system $\mathcal{D}_{n, \mathcal{C}}$ is a constant BT over $\mathcal{C}=\mathcal{C}_{0}$ isomorphic to $G_{\mathcal{C}}$ for some BT $G$ over $\operatorname{Spec}(l)$, the base of the induction holds for $t=0$. As $G_{\mathcal{C}_{t+1}}$ is a canonical lift of $G_{\mathcal{C}_{t}}$ to $\mathcal{C}_{t+1}$, the lifts of $G_{\mathcal{C}_{t}}$ to BTs over $\mathcal{C}_{t+1}$ are parametrized by the global sections of an $\mathcal{O}_{\mathcal{C}}$-module isomorphic to $\left(\mathcal{I}^{t+1} / \mathcal{I}^{t+2}\right)^{e_{G}}$, where $e_{G}$ is the product of the dimension and the codimension of $G$. From this and the previous paragraph, we get that $G_{\mathcal{C}_{t+1}}$ is the only lift of $G_{\mathcal{C}_{t}}$ to a BT over $\mathcal{C}_{t+1}$. Thus, if the inductive system $\mathcal{D}_{n, \mathcal{C}_{t}}$ is a constant BT over $\mathcal{C}_{t}$ isomorphic to $G_{\mathcal{C}_{t}}$, then the inductive system $\mathcal{D}_{n, \mathcal{C}_{t+1}}$ is also a constant BT over $\mathcal{C}_{t+1}$ isomorphic to $G_{\mathcal{C}_{t+1}}$. This ends the inductive step and thus also the induction.

From the previous paragraph, we get that the BT $\mathcal{D} \widehat{\mathbb{P}_{l}^{N}}$ over the formal completion $\widehat{\mathbb{P}_{l}^{N}}$ of $\mathbb{P}_{l}^{N}$ along $\mathcal{C}$ induced naturally by the inductive system $\mathcal{D}_{n, \mathcal{U}_{\infty}}$ is also constant isomorphic to $G_{\overparen{\mathbb{P}_{l}^{N}}}$.

We now consider the case where $\mathcal{C} \neq \mathcal{C}^{\mathrm{n}}$. Let $\varepsilon: \mathcal{C}^{\mathrm{n}} \rightarrow \mathbb{P}_{l}^{N} \times_{\operatorname{Spec}(l)} \mathbb{P}_{l}^{3}$ be a closed embedding whose projections are the composite morphism $\mathcal{C}^{\mathrm{n}} \rightarrow \mathcal{C} \rightarrow \mathbb{P}_{l}^{N}$ and a closed embedding $\mathcal{C}^{\mathrm{n}} \rightarrow \mathbb{P}_{l}^{3}$. Let $\mathcal{J}$ be the ideal sheaf of $\mathcal{O}_{\mathbb{P}_{l}^{N} \times \operatorname{Spec}(l)} \mathbb{P}_{l}^{3}$ which defines $\mathcal{C}^{\text {n }}$. We have a commutative diagram

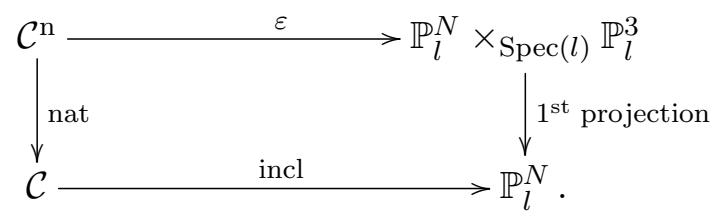

It is easy to see that for each $t \in \mathbb{N}^{*}$, the $t$ th symbolic power $\mathcal{I}^{(t)}$ of $\mathcal{I}$ (in the sense of [ZS58, Chapter IV, Definition of Section 12]) is the largest $\mathcal{O}_{\mathbb{P}_{l}^{N}}$-submodule of $\mathcal{I}$ that maps naturally into $\mathcal{J}^{t}$. As above, we argue that the $t$ th symmetric power $S^{t}\left(\mathcal{J} / \mathcal{J}^{2}\right)=\mathcal{J}^{t} / \mathcal{J}^{t+1}$ has no non-zero global section. From this and the natural inclusion $\mathcal{I}^{(t)} / \mathcal{I}^{(t+1)} \rightarrow$ nat $_{*}\left(\mathcal{J}^{t} / \mathcal{J}^{t+1}\right)$ of $\mathcal{O}_{\mathcal{C}}$-modules, we get that $\mathcal{I}^{(t)} / \mathcal{I}^{(t+1)}$ also has no non-zero global section.

As the inductive system $\mathcal{D}_{n, \mathcal{C}}$ is a constant BT over $\mathcal{C}$ isomorphic to $G_{\mathcal{C}}$, an induction on $t \in \mathbb{N}$ similar to the one above shows that the BT given by the inductive system $\mathcal{D}_{n, \mathcal{C}_{(t)}}$ over the $t$ th symbolic infinitesimal neighborhood $\mathcal{C}_{(t)}$ of $\mathcal{C}$ in $\mathbb{P}_{l}^{N}$ (defined by the ideal sheaf $\mathcal{I}^{(t+1)}$ of $\mathbb{P}_{l}^{N}$ ) is a constant BT over $\mathcal{C}_{(t)}$ isomorphic to $G_{\mathcal{C}_{(t)}}$, compatibly for different values of $t$.

By [ZS60, Chapter VIII, Corollary 5 of Theorem 13] applied to inclusions $\mathcal{I}(U) \subset \mathcal{O}_{\mathbb{P}_{l}^{N}}(U)$ for finitely many affine open subschemes $U$ of $\mathbb{P}_{l}^{N}$, we have that for each $t \in \mathbb{N}^{*}$, there exists an 


\section{O. GabBer And A. VAsiu}

integer $q \geqslant t$ such that we have $\mathcal{I}^{(q)} \subset \mathcal{I}^{t}$. This implies that

$$
\lim _{t \in \mathbb{N}^{*}} \mathcal{O}_{\mathbb{P}_{l}^{N}} / \mathcal{I}^{t}=\lim _{t \in \mathbb{N}^{*}} \mathcal{O}_{\mathbb{P}_{l}^{N}} / \mathcal{I}^{(t)}
$$

From this and the previous paragraph, we get that the BT $\mathcal{D}_{\widehat{\mathbb{P}_{l}^{N}}}$ over the formal completion $\widehat{\mathbb{P}_{l}^{N}}$ of $\mathbb{P}_{l}^{N}$ along $\mathcal{C}$ induced naturally by the inductive system $\mathcal{D}_{n, \mathcal{U}_{\infty}}$ is also constant and isomorphic to $G \widehat{\mathbb{P}_{l}^{N}}$.

Remark 5.2. The fact that $H^{0}\left(\mathcal{C}, \mathcal{J}^{(t)} / \mathcal{J}^{(t+1)}\right)=0$ for $t>0$ can be proved without using $\mathcal{C}^{\text {n }}$ as follows. Let $\mathcal{Z}$ be an integral closed scheme of a smooth $k$-scheme $\mathcal{X}$, let $\mathcal{J}$ be the ideal sheaf defining $\mathcal{Z}$ in $\mathcal{X}$, let $\mathcal{J}^{(t)}$ be the $t$ th symbolic power, and let Diff be the sheaf of differential operators on $\mathcal{X}$ in the sense of [GD67, Section 16.8], filtered by $\operatorname{Diff}^{n}=\operatorname{Diff}_{\mathcal{X} / \operatorname{Spec}(k)}^{n}(n \in \mathbb{N})$. Then $\operatorname{Diff}^{n}\left(\mathcal{J}^{(t)}\right) \subset \mathcal{J}^{(t-n)}\left(\mathcal{J}^{(m)}:=\mathcal{O}_{\mathcal{X}}\right.$ for $\left.m \leqslant 0\right)$, and this induces Difft $/$ Diff $^{t-1} \otimes_{\mathcal{O}_{\mathcal{X}}} \mathcal{J}^{(t)} / \mathcal{J}^{(t+1)}=$ $\underline{\operatorname{Hom}}\left(\operatorname{Sym}^{t}\left(\Omega_{\mathcal{X} / k}^{1}\right), \mathcal{O}_{\mathcal{X}}\right) \rightarrow \mathcal{O}_{\mathcal{Z}}$, hence $\mathcal{J}^{(t)} / \mathcal{J}^{(t+1)} \rightarrow \operatorname{Sym}^{t}\left(\Omega_{\mathcal{X} / k}^{1}\right) \otimes_{\mathcal{O}_{\mathcal{X}}} \mathcal{O}_{\mathcal{Z}}$, which is injective if $\mathcal{Z}$ is generically smooth. For $\mathcal{X}$ a projective space and $\mathcal{Z}$ of positive dimension and generically smooth, this proves that $H^{0}\left(\mathcal{Z}, \mathcal{J}^{(t)} / \mathcal{J}^{(t+1)}\right)=0$ for $t>0$.

To continue the proof of Theorem 1.2, in this paragraph we consider the particular case where $l^{\prime}=l$ is an algebraically closed field. This assumption implies that $\mathcal{C}$ is G3 in $\mathbb{P}_{l}^{N}$, as recalled in Section 4. From this and Lemma 4.1 applied with $(\mathcal{U}, \mathcal{V})=\left(\mathcal{U}_{n}, \mathcal{V}_{n}\right)$, where $\mathcal{V}_{n}$ is the coherent locally free $\mathcal{O}_{\mathcal{U}_{n}}$-algebra that defines $\mathcal{D}_{n}$, we get a natural ring identification $H^{0}\left(\mathcal{U}_{n}, \mathcal{V}_{n}\right)=H^{0}\left(\widehat{\mathbb{P}_{l}^{N}}, \widehat{\mathcal{V}_{n}}\right)$, where $\widehat{\mathcal{V}_{n}}:=\chi^{*}\left(\mathcal{V}_{n}\right)$ is the formal completion of $\mathcal{V}_{n}$ along $\mathcal{C}$. From Proposition 5.1, we get that $H^{0}\left(\widehat{\mathbb{P}_{l}^{N}}, \widehat{\mathcal{V}_{n}}\right)$ is canonically identified with the ring of regular functions on $G\left[p^{n}\right]$. We have similar identifications for $\mathcal{V}_{n} \otimes_{\mathcal{O}_{\mathcal{u}_{n}}} \mathcal{V}_{n}, \widehat{\mathcal{V}_{n}} \otimes_{\mathcal{O}_{\overparen{\mathbb{P}_{l}^{N}}}} \widehat{\mathcal{V}_{n}}$ and $G\left[p^{n}\right] \times_{\operatorname{Spec}(l)} G\left[p^{n}\right]$, with certain compatibilities. Therefore, we have a canonical homomorphism $\vartheta_{n}: \mathcal{D}_{n} \rightarrow G\left[p^{n}\right] \mathcal{U}_{n}$ of finite flat group schemes whose pullback to the formal completion $\widehat{\mathbb{P}_{l}^{N}}$ is an isomorphism, being the truncation of level $n$ of an isomorphism as in Proposition 5.1. From this and the first part of Fact 2.1(d), we get that $\vartheta_{n}$ is an isomorphism over an open subscheme $\mathcal{W}_{n}$ of $\mathcal{U}_{n}$ which contains $\mathcal{C}$. As the complement of $\mathcal{W}_{n}$ in $\mathbb{P}_{l}^{N}$ has codimension at least 2 in $\mathbb{P}_{l}^{N}$, from the second part of Fact 2.1(d), we get that, in fact, $\vartheta_{n}$ is an isomorphism. The isomorphisms $\vartheta_{n}$ are compatible in the sense that for all $n, m \in \mathbb{N}^{*}$, we have $\vartheta_{n+m}\left[p^{n}\right]=\vartheta_{n, \mathcal{U}_{n+m}}$. Therefore, $\mathcal{D}_{n}$ extends uniquely (up to unique isomorphism) to a constant $\mathrm{BT}_{n} \mathcal{D}_{n}^{+}$over $\mathbb{P}_{l}^{N}$ isomorphic to $G\left[p^{n}\right]_{\mathbb{P}_{l}^{N}}$. Due to the mentioned compatibility of the isomorphisms $\vartheta_{n}$ and the uniqueness of the extension $\mathcal{D}_{n}^{+}$of $\mathcal{D}_{n}$, we have canonical identifications $\mathcal{D}_{n+1}^{+}\left[p^{n}\right]=\mathcal{D}_{n}^{+}$. Thus the inductive system $\mathcal{D}_{n}^{+}$is a constant BT over $\mathbb{P}_{l}^{N}$ isomorphic to $G_{\mathbb{P}_{l}^{N}}$. Moreover, $G\left[p^{n}\right]$ is the affine group scheme over $\operatorname{Spec}(l)$ defined by the Hopf $l$-algebra of global functions on $\mathcal{D}_{n}^{+}$and thus also on either $\mathcal{D}_{n}$ or $\mathcal{D}_{n, \mathcal{U}_{n+m}}$; use Fact 2.1(c) and the fact that the complement of $\mathcal{U}_{n}$ in $\mathbb{P}_{l}^{N}$ has codimension at least 2 in $\mathbb{P}_{l}^{N}$.

We now consider the general case. By enlarging $l^{\prime}$, we can assume that it is an algebraically closed field. Let $n, m \in \mathbb{N}^{*}$. If $\omega_{n}: \mathcal{D}_{n} \rightarrow \mathcal{U}_{n}$ is the structure morphism, then the $\mathcal{O}_{U_{n}}$-linear map $\mathcal{O}_{\mathcal{U}_{n}} \otimes_{l} H^{0}\left(\mathcal{U}_{n}, \omega_{n, *}\left(\mathcal{O}_{\mathcal{D}_{n}}\right)\right) \rightarrow \omega_{n, *}\left(\mathcal{O}_{\mathcal{D}_{n}}\right)$ is an isomorphism (as the base change to $\mathcal{U}_{n, l^{\prime}}$ is), and thus the Hopf $\mathcal{O}_{U_{n}}$-algebra structure on $\omega_{n, *}\left(\mathcal{O}_{\mathcal{D}_{n}}\right)$ defines a commutative and cocommutative Hopf $l$-algebra structure on $H^{0}\left(\mathcal{U}_{n}, \omega_{n, *}\left(\mathcal{O}_{\mathcal{D}_{n}}\right)\right)$ and hence a finite group scheme $G_{n}$ over $l$ and a canonical isomorphism $\mathcal{D}_{n} \rightarrow G_{n, \mathcal{U}_{n}}$. This implies that $G_{n}$ is a $\operatorname{BT}_{n}$ over $\operatorname{Spec}(l)$. The canonical closed embedding homomorphisms $\mathcal{D}_{n, \mathcal{U}_{n+m}} \rightarrow \mathcal{D}_{n+m}$ and the canonical epimorphisms $\mathcal{D}_{n+m} \rightarrow$ $\mathcal{D}_{n, \mathcal{U}_{n+m}}$ induce naturally homomorphisms $G_{n} \rightarrow G_{n+m}$ and $G_{n+m} \rightarrow G_{n}$ which are closed 


\section{Purity for BARsotti-TATE Groups}

embeddings and epimorphisms, respectively, as their extensions to $l^{\prime}$ are so by the previous paragraph. By similar verifications, these last homomorphisms define a BT $G$ over $\operatorname{Spec}(l)$ such that for all $n \in \mathbb{N}^{*}$, we have $G_{n}=G\left[p^{n}\right]$. We conclude that for each $n \in \mathbb{N}^{*}$, the $\mathrm{BT}_{n} \mathcal{D}_{n}$ extends uniquely (up to unique isomorphism) to a constant $\mathrm{BT}_{n} \mathcal{D}_{n}^{+}$over $\mathbb{P}_{l}^{N}$ isomorphic to $G\left[p^{n}\right]_{\mathbb{P}_{l}^{N}}$ and the identification $\mathcal{D}_{n+1}\left[p^{n}\right]=\mathcal{D}_{n, \mathcal{U}_{n+1}}$ extends to an identification $\mathcal{D}_{n+1}^{+}\left[p^{n}\right]=\mathcal{D}_{n}^{+}$which is the pullback to $\mathbb{P}_{l}^{N}$ of the identification $\left(G\left[p^{n+1}\right]\right)\left[p^{n}\right]=G\left[p^{n}\right]$ over $\operatorname{Spec}(l)$. Thus Theorem 1.2 holds.

\section{Proof of Theorem 1.1(b)}

If $d=2$, then $R$ admits a faithfully flat regular local extension of the form $W(l)\left[\left[x_{1}, x_{2}\right]\right] /(h)$ with $l$ a perfect field which contains $k$ and is such that $\bar{h} \notin\left(x_{1}^{p}, x_{2}^{p}, x_{1}^{p-1} x_{2}^{p-1}\right)$; see Fact 3.1. Thus $R$ is $p$-quasi-healthy by [VZ10, Theorem 3].

In the rest of this section, we will prove by induction on $d \geqslant 3$ that Theorem 1.1(b) also holds for $d \geqslant 3$. The base of the induction (that is, the case $d=3$ ) is checked in Subsection 6.1. The inductive step (that is, the passage from $d-1 \geqslant 3$ to $d \geqslant 4$ ) is checked in Subsection 6.2.

Let $D_{U}$ be a BT over the punctured spectrum $U$ of a regular local scheme $X=\operatorname{Spec}(R)$ of mixed characteristic $(0, p)$ and dimension $d \geqslant 3$. We have to show that if condition ( $q$ ) holds for $R$, then $D_{U}$ extends to a BT $D$ over $X$. Based on Lemma 2.6 and Fact 3.1, by passing to a faithfully flat extension, we can assume that $R$ is also complete of the form $R=W(k)\left[\left[x_{1}, \ldots, x_{d}\right]\right] /(h)$, with $k$ an algebraically closed field of positive characteristic $p$ and with $h \in W(k)\left[\left[x_{1}, \ldots, x_{d}\right]\right]$ such that $\bar{h} \notin\left(x_{1}^{p}, \ldots, x_{d}^{p}\right)+\left(x_{1}, \ldots, x_{d}\right)^{2 p-2}$. For $i \in\{1, \ldots, d\}$, let $y_{i}:=x_{i}+(h) \in R$. We can also assume that the residue field $k$ of $R$ is uncountable and (as in Section 3) that $y_{1}, \ldots, y_{d}$ is a regular system of parameters of $R$.

\subsection{The base of the induction: The case $d=3$}

In this subsection, we will assume that $d=3$. Thus $R=W(k)\left[\left[x_{1}, x_{2}, x_{3}\right]\right] /(h)$ has $y_{1}, y_{2}, y_{3}$ as a regular system of parameters of $R$. Let $Z$ be the blowup of $X$ along its closed point; it is a regular scheme of dimension 3 which is projective over $X$ and which is the union of the open subscheme $U$ and a closed subscheme $\mathbb{P}_{k}^{2}$. From Proposition 2.4, we get that the restriction of $D_{U}$ to the generic point of $Z$ (that is, to $\operatorname{Spec}(\operatorname{Frac}(R))$ ) extends to a $\mathrm{BT} D_{\mathrm{Spec}(O)}$ over the spectrum of the discrete valuation ring $O$ which is the local ring in $Z$ of the generic point of $\mathbb{P}_{k}^{2}$. From this and Fact 2.1(b), we get that for each $n \in \mathbb{N}^{*}$, there exists a finite set $\mathcal{S}_{n}$ of closed points of $\mathbb{P}_{k}^{2}$ such that the $\mathrm{BT}_{n} D_{U}\left[p^{n}\right]$ over $U$ extends uniquely (up to unique isomorphism) to a finite flat group scheme $E_{n}$ over $Z \backslash \mathcal{S}_{n}$ whose pullback to $\operatorname{Spec}(O)$ is $D_{\operatorname{Spec}(O)}\left[p^{n}\right]$.

Recall the standard fact that if $\triangle$ is a finite locally free commutative group scheme annihilated by $p^{n}$ over a scheme $\Sigma$, then the set of points $z$ of $\Sigma$ such that the fiber of $\triangle$ at $z$ is a $\mathrm{BT}_{n}$ over the residue field of $z$ is open and $\triangle$ is a $\mathrm{BT}_{n}$ over the corresponding open subscheme. Thus let $\mathcal{K}_{n}$ be the smallest closed subset of $\mathbb{P}_{k}^{2}$ which contains $\mathcal{S}_{n}$ and is such that the restriction of $E_{n}$ to $Z \backslash \mathcal{K}_{n}$ is a $\mathrm{BT}_{n}$; the dimension of $\mathcal{K}_{n}$ is at most 1 .

We can assume that we have a chain of inclusions $\mathcal{S}_{1} \subset \mathcal{S}_{2} \subset \cdots \subset \mathcal{S}_{n} \subset \cdots$. Thus we have a second chain of inclusions $\mathcal{K}_{1} \subset \mathcal{K}_{2} \subset \cdots \subset \mathcal{K}_{n} \subset \cdots$ such that for each $n \in \mathbb{N}^{*}$, the $\mathrm{BT}_{n} \mathrm{~s}$ $E_{n+1}\left[p^{n}\right]_{Z \backslash \mathcal{K}_{n+1}}$ and $E_{n, Z \backslash \mathcal{K}_{n+1}}$ coincide over $Z \backslash \mathcal{K}_{n+1}$. The set $\mathcal{S}_{\infty}:=\cup_{n \geqslant 1} \mathcal{S}_{n}$ is countable, and the ind-constructible set $\mathcal{K}_{\infty}:=\cup_{n \geqslant 1} \mathcal{K}_{n}$ has a countable number of maximal points and is the union of their closures. ${ }^{3}$ Moreover, $D_{U}$ extends to a BT $D_{Z \backslash \mathcal{K}_{\infty}}$ over the stable under

\footnotetext{
${ }^{3}$ Recall that a point of a topological space is said to be a maximal point if its closure is not strictly contained
} 


\section{O. Gabber and A. Vasiu}

generization, pro-constructible subset $Z \backslash \mathcal{K}_{\infty}=\cap_{n=1}^{\infty}\left(Z \backslash \mathcal{K}_{n}\right)$ of $Z$ (see Remark 1.3).

Claim 6.1. For each $n \in \mathbb{N}^{*}$, the set $\mathcal{K}_{n}$ is finite, and therefore (by enlarging $\mathcal{S}_{n}$ ) we can assume that $\mathcal{S}_{n}=\mathcal{K}_{n}$.

We begin the proof of Claim 6.1 by introducing notation pertaining to $h$.

6.1.1 On $\bar{h} \in k\left[\left[x_{1}, x_{2}, x_{3}\right]\right]$. For each integer $i \in\{1, \ldots, 2 p-3\}$, let $\mathbb{J}_{i}^{3}$ be the subset of $\{0, \ldots, p-1\}^{3}$ formed by those triples whose sum is $i$. We write

$$
\bar{h}\left(x_{1}, x_{2}, x_{3}\right)=\bar{h}_{0}\left(x_{1}, x_{2}, x_{3}\right)+\sum_{i=1}^{2 p-3} \bar{h}_{i}\left(x_{1}, x_{2}, x_{3}\right),
$$

where $\bar{h}_{0}$ belongs to the ideal $\left(x_{1}^{p}, x_{2}^{p}, x_{3}^{p}\right)+\left(x_{1}, x_{2}, x_{3}\right)^{2 p-2}$ of $k\left[\left[x_{1}, x_{2}, x_{3}\right]\right]$ and where each

$$
\bar{h}_{i}\left(x_{1}, x_{2}, x_{3}\right)=\sum_{\left(i_{1}, i_{2}, i_{3}\right) \in \mathbb{J}_{i}^{3}} \delta_{i_{1}, i_{2}, i_{3}} x_{1}^{i_{1}} x_{2}^{i_{2}} x_{3}^{i_{3}} \in k\left[x_{1}, x_{2}, x_{3}\right]
$$

is a homogeneous polynomial of degree $i$. As $\bar{h} \notin\left(x_{1}^{p}, x_{2}^{p}, x_{3}^{p}\right)+\left(x_{1}, x_{2}, x_{3}\right)^{2 p-2}$, there exists a smallest element $c \in\{1, \ldots, 2 p-3\}$ such that we have $\bar{h}_{c} \neq 0$.

\subsubsection{Good regular closed subschemes of $X$ of dimension 2. For each triple}

$$
\zeta=\left(\zeta_{1}, \zeta_{2}, \zeta_{3}\right) \in W(k)^{3}
$$

such that its reduction $\bar{\zeta}=\left(\bar{\zeta}_{1}, \bar{\zeta}_{2}, \bar{\zeta}_{3}\right)$ modulo $p$ is a non-zero element of $k^{3}$, we consider the regular closed subscheme $\operatorname{Spec}\left(S_{\zeta}\right)$ of $X$, where $S_{\zeta}:=R /\left(\zeta_{1} y_{1}+\zeta_{2} y_{2}+\zeta_{3} y_{3}\right)$. Let $Z_{\zeta}$ be the closed subscheme of $Z$ which is the blowup of $\operatorname{Spec}\left(S_{\zeta}\right)$ along its closed point; it is the union of its open subscheme $U \cap \operatorname{Spec}\left(S_{\zeta}\right)$ and a $\mathbb{P}_{k, \zeta}^{1}$ curve inside $\mathbb{P}_{k}^{2}$. It is easy to see that, with respect to the usual projective coordinates $\left[w_{0}, w_{1}, w_{2}\right]$ of $\mathbb{P}_{k}^{2}$, the curve $\mathbb{P}_{k, \zeta}^{1}$ of $\mathbb{P}_{k}^{2}$ is defined by the equation $\bar{\zeta}_{1} w_{0}+\bar{\zeta}_{2} w_{1}+\bar{\zeta}_{3} w_{2}=0$. If $P=\left[\gamma_{0}, \gamma_{1}, \gamma_{2}\right] \in \mathcal{S}_{\infty} \subset \mathbb{P}_{k}^{2}$ with $\gamma_{0}, \gamma_{1}, \gamma_{2} \in k$ not all zero, then condition $P \notin \mathbb{P}_{k, \zeta}^{1}$ gets translated into the inequality $\bar{\zeta}_{1} \gamma_{0}+\bar{\zeta}_{2} \gamma_{1}+\bar{\zeta}_{3} \gamma_{2} \neq 0$. Thus there exists a countable union $\mathcal{L}_{\infty}$ of lines in $\mathbb{P}_{k}^{2}$ such that we have $\mathbb{P}_{k, \zeta}^{1} \cap \mathcal{S}_{\infty}=\emptyset$ if and only if $\left[\bar{\zeta}_{1}, \bar{\zeta}_{2}, \bar{\zeta}_{3}\right] \notin \mathcal{L}_{\infty}(k)$

Let $\mathcal{P}_{\infty}$ be the countable subset of $\mathbb{P}_{k}^{2}(k)$ such that $\mathbb{P}_{k, \zeta}^{1}$ is not contained in $\mathcal{K}_{\infty}$ if and only if $\left[\bar{\zeta}_{1}, \bar{\zeta}_{2}, \bar{\zeta}_{3}\right] \notin \mathcal{P}_{\infty}$

From Lemma 3.2 applied with $(l, d)=(k, 3)$, we get that there exists an open dense subscheme $\mathbb{O}$ of $\mathbb{A}_{k}^{2}$ such that for each pair $\left(v_{1}, v_{2}\right) \in \mathbb{O}(k) \subset k^{2}$, the polynomial

$$
\bar{h}_{c, \zeta}\left(x_{1}, x_{2}\right):=\bar{h}_{c}\left(x_{1}, x_{2}, v_{1} x_{1}+v_{2} x_{2}\right)
$$

does not belong to the ideal $\left(x_{1}^{p}, x_{2}^{p}, x_{1}^{p-1} x_{2}^{p-1}\right)$ of $k\left[\left[x_{1}, x_{2}\right]\right]$. If $\left(\bar{\zeta}_{1}, \bar{\zeta}_{2}, \bar{\zeta}_{3}\right)=\left(-v_{1},-v_{2}, 1\right)$ with $\left(v_{1}, v_{2}\right) \in \mathbb{O}(k)$, then $S_{\zeta}=R /\left(\zeta_{1} y_{1}+\zeta_{2} y_{2}+\zeta_{3} y_{3}\right)$ is a regular ring isomorphic to $W(k)\left[\left[x_{1}, x_{2}\right]\right] /\left(h_{\zeta}\right)$, where

$$
h_{\zeta}\left(x_{1}, x_{2}\right):=h\left(x_{1}, x_{2},-\zeta_{3}^{-1} \zeta_{1} x_{1}-\zeta_{3}^{-1} \zeta_{2} x_{2}\right) \in W(k)\left[\left[x_{1}, x_{2}\right]\right]
$$

is such that its reduction $\bar{h}_{\zeta}$ modulo $p$ has $\bar{h}_{c, \zeta}$ as its homogeneous component of degree $c$, and therefore it does not belong to the ideal $\left(x_{1}^{p}, x_{2}^{p}, x_{1}^{p-1} x_{2}^{p-1}\right)$ of $k\left[\left[x_{1}, x_{2}\right]\right]$; thus in such a case, $S_{\zeta}$ is of mixed characteristic $(0, p)$ and is $p$-quasi-healthy (by [VZ10, Theorem 3]).

in the closure of another point. For sober spaces, the maximal points are the generic points of the irreducible components. 


\section{Purity For BARsotti-TATE GROUPS}

We identify $\mathbb{A}_{k}^{2}$ with an open subscheme of $\mathbb{P}_{k}^{2}$ via the embedding $\left(v_{1}, v_{2}\right) \mapsto\left[-v_{1},-v_{2}, 1\right]$. As $k$ is uncountable, the set of all closed points of the open dense subscheme $\mathbb{O}$ of $\mathbb{A}_{k}^{2} \subset \mathbb{P}_{k}^{2}$ cannot be contained in $\mathcal{L}_{\infty}(k) \cup \mathcal{P}_{\infty}$; that is, there exist pairs $\left(v_{1}, v_{2}\right) \in \mathbb{O}(k)$ such that we have $\left[-v_{1},-v_{2}, 1\right] \notin \mathcal{L}_{\infty}(k) \cup \mathcal{P}_{\infty}$. If $\left(v_{1}, v_{2}\right)$ is such a pair, then by choosing $\left(\bar{\zeta}_{1}, \bar{\zeta}_{2}, \bar{\zeta}_{3}\right)=\left(-v_{1},-v_{2}, 1\right)$, we get that the regular closed subscheme $\operatorname{Spec}\left(S_{\zeta}\right)$ of $X$ is good in the following sense:

$(\sharp)$ The regular ring $S_{\zeta}$ is $p$-quasi-healthy, and moreover the line $\mathbb{P}_{k, \zeta}^{1}$ neither intersects the countable subset $\mathcal{S}_{\infty}$ of $\mathbb{P}_{k}^{2}$ nor is contained in $\mathcal{K}_{\infty}$.

6.1.3 Proof of Claim 6.1. As $S_{\zeta}$ is p-quasi-healthy by $(\sharp)$, the restriction of $D_{U}$ to $U \cap$

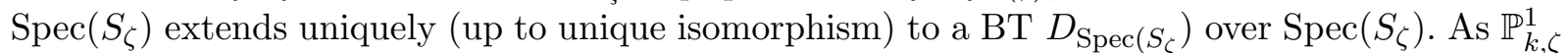
does not intersect $\mathcal{S}_{n}$ by $(\sharp)$, for $n \in \mathbb{N}^{*}$ we can speak about the pullback $E_{n, \zeta}$ of $E_{n}$ to $Z_{\zeta}$. Let $E_{n, O_{\zeta}}$ be the restriction of $E_{n, \zeta}$ to the spectrum of the local ring $O_{\zeta}$ of $Z_{\zeta}$ which is a discrete valuation ring that dominates $S_{\zeta}$. As $\mathbb{P}_{k, \zeta}^{1}$ is not contained in $\mathcal{K}_{\infty}$, the inductive system $E_{n, O_{\zeta}}$ is a BT over $\operatorname{Spec}\left(O_{\zeta}\right)$ which, based on Tate's extension theorem, is the pullback of $D_{\operatorname{Spec}\left(S_{\zeta}\right)}$ to $\operatorname{Spec}\left(O_{\zeta}\right)$. This implies that for each $n \in \mathbb{N}^{*}$, the finite flat group scheme $E_{n, \zeta}$ is the pullback to $Z_{\zeta}$ of $D_{\mathrm{Spec}\left(S_{\zeta}\right)}\left[p^{n}\right]$. Thus the inductive system $E_{n, \zeta}$ is a BT over $Z_{\zeta}$. This implies that $Z_{\zeta}$, and therefore also $\mathbb{P}_{k, \zeta}^{1}$, does not intersect $\mathcal{K}_{\infty}$. As two irreducible projective curves in $\mathbb{P}_{k}^{2}$ always intersect and as $\mathbb{P}_{k, \zeta}^{1} \cap \mathcal{K}_{n}=\emptyset$, we conclude that each $\mathcal{K}_{n}$ has dimension at most 0 , and therefore by enlarging $\mathcal{S}_{n}$, we can assume that for each $n \in \mathbb{N}^{*}$, we have $\mathcal{S}_{n}=\mathcal{K}_{n}$. Thus also $\mathcal{K}_{\infty}=\mathcal{S}_{\infty}$, and each $E_{n}$ is a $\mathrm{BT}_{n}$ over $Z \backslash \mathcal{S}_{n}$. This ends the proof of Claim 6.1.

6.1.4 Applying Theorem 1.2. Let $G$ be the fiber of $D_{\operatorname{Spec}\left(S_{\zeta}\right)}$ over the closed point $\operatorname{Spec}(k)$ of $\operatorname{Spec}\left(S_{\zeta}\right)$. For $n \in \mathbb{N}^{*}$, as $E_{n, \zeta}$ is the pullback of $D_{\operatorname{Spec}\left(S_{\zeta}\right)}\left[p^{n}\right]$ to $Z_{\zeta}$, the pullback of $E_{n}$ to $\mathbb{P}_{k, \zeta}^{1}$ is canonically identified with $G\left[p^{n}\right]_{\mathbb{P}_{k, \zeta}^{1}}$. From this and Theorem 1.2 applied with $\left(l, N, \mathcal{C}, \mathcal{U}_{n}, \mathcal{D}_{n}\right)$ equal to $\left(k, 2, \mathbb{P}_{k, \zeta}^{1}, \mathbb{P}_{k}^{2} \backslash \mathcal{K}_{n}, E_{n, \mathbb{P}_{k}^{2} \backslash \mathcal{K}_{n}}\right)$, we get that the restriction $D_{\mathbb{P}_{k}^{2} \backslash \mathcal{S}_{\infty}}$ of $D_{Z \backslash \mathcal{S}_{\infty}}$ to $\mathbb{P}_{k}^{2} \backslash \mathcal{S}_{\infty}$ is isomorphic to $G_{\mathbb{P}_{k}^{2} \backslash \mathcal{S}_{\infty}}$, and thus it extends to a constant BT $D_{\mathbb{P}_{k}^{2}}$ over $\mathbb{P}_{k}^{2}$ isomorphic to $G_{\mathbb{P}_{k}^{2}}$.

6.1.5 Liftings to infinitesimal neighborhoods of $\mathbb{P}_{k}^{2}$ in $Z$. Let $\mathfrak{m}:=\left(y_{1}, y_{2}, y_{3}\right)$ be the maximal ideal of $R$. As $\mathfrak{m} \mathcal{O}_{Z}$ is the ideal sheaf of $\mathcal{O}_{Z}$ that defines the closed subscheme $\mathbb{P}_{k}^{2}$ of $Z$, for each $t \in \mathbb{N}$, for the invertible $\mathcal{O}_{\mathbb{P}_{k}^{2}}$-module $\mathfrak{m}^{t} \mathcal{O}_{Z} / \mathfrak{m}^{t+1} \mathcal{O}_{Z} \simeq \mathcal{O}_{\mathbb{P}_{k}^{2}}(t)$ we have

$$
H^{1}\left(Z, \mathfrak{m}^{t} \mathcal{O}_{Z} / \mathfrak{m}^{t+1} \mathcal{O}_{Z}\right)=H^{1}\left(\mathbb{P}_{k}^{2}, \mathfrak{m}^{t} \mathcal{O}_{Z} / \mathfrak{m}^{t+1} \mathcal{O}_{Z}\right)=0
$$

We consider a coherent locally free module $\mathcal{V}_{t+1}$ of rank $r$ over the $(t+1)$ th infinitesimal neighborhood $\mathbb{P}_{k, t+1}^{2}$ of $\mathbb{P}_{k}^{2}$ in $Z$ (that is, over the reduction modulo $\mathfrak{m}^{t+2} \mathcal{O}_{Z}$ of $Z$ ) such that the coherent locally free module $\mathcal{V}_{t}:=\mathcal{V}_{t+1} / \mathfrak{m}^{t+1} \mathcal{V}_{t+1}$ over the $t$ th infinitesimal neighborhood $\mathbb{P}_{k, t}^{2}$ of $\mathbb{P}_{k}^{2}$ in $Z$ is trivial and thus isomorphic to $\mathcal{O}_{\mathbb{P}_{k, t}^{2}}^{r}$. As we have

$\mathfrak{m}^{t+1} \mathcal{V}_{t+1}=\left(\mathfrak{m}^{t+1} \mathcal{O}_{Z} / \mathfrak{m}^{t+2} \mathcal{O}_{Z}\right) \otimes \mathcal{O}_{Z} \mathcal{V}_{t+1}=\left(\mathfrak{m}^{t+1} \mathcal{O}_{Z} / \mathfrak{m}^{t+2} \mathcal{O}_{Z}\right) \otimes \mathcal{O}_{Z} \mathcal{V}_{t}=\left(\mathfrak{m}^{t+1} \mathcal{O}_{Z} / \mathfrak{m}^{t+2} \mathcal{O}_{Z}\right)^{r}$

the short exact sequence

$$
0 \rightarrow \mathfrak{m}^{t+1} \mathcal{V}_{t+1} \rightarrow \mathcal{V}_{t+1} \rightarrow \mathcal{V}_{t} \rightarrow 0
$$

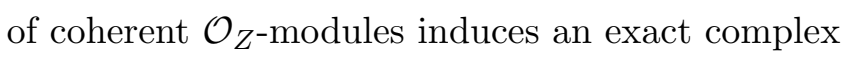

$$
H^{0}\left(Z, \mathcal{V}_{t+1}\right) \rightarrow H^{0}\left(Z, \mathcal{V}_{t}\right) \rightarrow H^{1}\left(Z, \mathfrak{m}^{t+1} \mathcal{O}_{Z} / \mathfrak{m}^{t+2} \mathcal{O}_{Z}\right)^{r}=0
$$

Therefore, the reduction homomorphism $H^{0}\left(Z, \mathcal{V}_{t+1}\right) \rightarrow H^{0}\left(Z, \mathcal{V}_{t}\right)$ is surjective, and this implies 


\section{O. Gabber and A. Vasiu}

that $\mathcal{V}_{t+1}$ is also a free $\mathcal{O}_{\mathbb{P}_{k, t+1}^{2}}$-module of rank $r$.

By induction on $t \in \mathbb{N}$, we check that the constant BT $D_{\mathbb{P}_{k}^{2}}$ over $\mathbb{P}_{k}^{2}$ lifts uniquely (up to unique isomorphism) to a BT $D_{\mathbb{P}_{k, t}^{2}}$ over $\mathbb{P}_{k, t}^{2}$ in such a way that its restriction to the reduction modulo $\mathfrak{m}^{t+1}$ of $Z \backslash \mathcal{S}_{\infty}$ is induced naturally by $D_{Z \backslash \mathcal{S}_{\infty}}$, and, moreover, the coherent $\mathcal{O}_{\mathbb{P}_{k, t}^{2}}$-module associated naturally to the structure sheaf of the $\mathrm{BT}_{n} D_{\mathbb{P}_{k, t}^{2}}\left[p^{n}\right]$ is free for all $n \in \mathbb{N}^{*}$ (in particular, for depth reasons each $D_{\mathbb{P}_{k, t}^{2}}\left[p^{n}\right]$ extends the reduction of $E_{n}$ modulo $\mathfrak{m}^{t+1}$ ).

The case $t=0$ was accomplished in Paragraph 6.1.4. The passage from $t$ to $t+1$ goes as follows. From Grothendieck-Messing deformation theory, we get that there exists a torsor $\mathcal{T}_{t}$

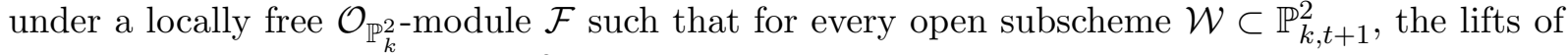
the BT $D_{\mathbb{P}_{k, t}^{2} \cap \mathcal{W}}:=D_{\mathbb{P}_{k, t}^{2}} \times_{\mathbb{P}_{k, t}^{2}}\left(\mathbb{P}_{k, t}^{2} \cap \mathcal{W}\right)$ to BTs over $\mathcal{W}$ are parametrized by the global sections $\mathcal{T}_{t}(\mathcal{W})$. The automorphism groups of such lifts are trivial. Similarly, for each integer $n \geqslant 1$, if $\mathcal{W}$ is affine and contained in $Z \backslash \mathcal{S}_{n}$, the isomorphism classes of $\mathrm{BT}_{n}$ lifts of $E_{n} \times_{Z \backslash \mathcal{S}_{n}}\left(\mathbb{P}_{k, t}^{2} \cap \mathcal{W}\right)$ to $\mathcal{W}$ are parametrized by $\mathcal{T}_{t}(\mathcal{W})$ in a way compatible with $n$ and the previous sentence (see [Ill85, Théorème 4.4(c) and Corollaire 4.7]). By [Ill85, Théorème 4.4(c)], the automorphism groups of such lifts can be identified with $\mathcal{F}(\mathcal{W})$, and by [Ill85, Théorème 4.4(d)] the transition maps on such automorphism groups are trivial; that is, in the above situation for $n>1$, the automorphisms of a $\mathrm{BT}_{n}$ lift restrict to the trivial automorphism of the corresponding $\mathrm{BT}_{n-1}$ lift. Thus, for $\mathcal{W}$ a not necessarily affine open subscheme of $\mathbb{P}_{k, t+1}^{2} \backslash \mathcal{S}_{n}$, if we have a lift $\tilde{E}_{n}$ of $E_{n} \times{ }_{Z \backslash \mathcal{S}_{n}}\left(\mathbb{P}_{k, t}^{2} \cap \mathcal{W}\right)$ to a $\mathrm{BT}_{n}$ over $\mathcal{W}$, then there exists a uniquely determined BT lift $\tilde{D}$ of $D_{\mathbb{P}_{k, t}^{2} \cap \mathcal{W}}$ to $\mathcal{W}$ such that the lift $\tilde{D}\left[p^{n}\right]$ is locally isomorphic to $\tilde{E}_{n}$; for $n>1$, these local isomorphisms define a global isomorphism between the corresponding $\mathrm{BT}_{n-1}$ lifts. By limit arguments, we deduce that the lifts of $D_{Z \backslash \mathcal{S}_{\infty}}$ modulo $\mathfrak{m}^{t+1}$ to $Z \backslash \mathcal{S}_{\infty}$ modulo $\mathfrak{m}^{t+2}$ are parametrized by $H^{0}\left(\mathbb{P}_{k}^{2} \backslash \mathcal{S}_{\infty}, \mathcal{F}\right)=H^{0}\left(\mathbb{P}_{k}^{2}, \mathcal{F}\right)$, so we conclude that there exists a unique (up to unique isomorphism) BT over $\mathbb{P}_{k, t+1}^{2}$ which lifts $D_{\mathbb{P}_{k, t}^{2}}$ in such a way that its restriction to the reduction modulo $\mathfrak{m}^{t+2}$ of $Z \backslash \mathcal{S}_{\infty}$ is induced naturally by $D_{Z \backslash \mathcal{S}_{\infty}}$. By a previous argument, we get that the coherent $\mathcal{O}_{\mathbb{P}_{k, t+1}^{2}}$-module associated naturally to the structure sheaf of the $\mathrm{BT}_{n} D_{\mathbb{P}_{k, t+1}^{2}}\left[p^{n}\right]$ is free for all $n \in \mathbb{N}^{*}$. This ends the induction on $t \in \mathbb{N}$.

6.1.6 End of the proof in the case $d=3$. For $z \in \mathcal{S}_{n}$, let $\tau \in \mathcal{O}_{Z, z}$ be such that we have $\operatorname{Spec}\left(\mathcal{O}_{Z, z} /(\tau)\right)=\operatorname{Spec}\left(\mathcal{O}_{Z, z}\right) \times_{Z} \mathbb{P}_{k}^{2}$. As $\mathcal{O}_{Z, z}$ is a regular local ring of dimension $d=3$, for $t \in \mathbb{N}$ we have $\operatorname{depth}\left(\mathcal{O}_{Z, z} / \tau^{t+1} \mathcal{O}_{Z, z}\right)=d-1 \geqslant 2$, and thus for each coherent locally free $\mathcal{O}_{\mathbb{P}_{k, t}^{2}}$-module $\mathfrak{F}_{t}$, we have a canonical identification

$$
H^{0}\left(\mathbb{P}_{k, t}^{2} \backslash \mathcal{S}_{n}, \mathfrak{F}_{t}\right)=H^{0}\left(\mathbb{P}_{k, t}^{2}, \mathfrak{F}_{t}\right) .
$$

Thus the direct image via the open embedding $\mathbb{P}_{k, t}^{2} \backslash \mathcal{S}_{n} \rightarrow \mathbb{P}_{k, t}^{2}$ of the $\mathcal{O}_{\mathbb{P}_{k, t}^{2}} \backslash \mathcal{S}_{n}$-module associated to the reduction of $E_{n}$ modulo $\mathfrak{m}^{t+1}$ is the $\mathcal{O}_{\mathbb{P}_{k, t}^{2}}$-module associated to $D_{\mathbb{P}_{k, t}^{2}}\left[p^{n}\right]$. Based on this, from Lemma 2.7 applied to the local rings $\mathcal{O}_{Z, z}$, we get that for each $n \in \mathbb{N}^{*}$, the locally free $\mathcal{O}_{Z \backslash \mathcal{S}_{n}}$-module associated to $E_{n}$ extends (uniquely up to unique isomorphism) to a locally free $\mathcal{O}_{Z}$-module whose reduction modulo each $\mathfrak{m}^{t+1}$ is the $\mathcal{O}_{\mathbb{P}_{k, t}^{2}}$-module associated to $D_{\mathbb{P}_{k, t}^{2}}\left[p^{n}\right]$. This implies that each $E_{n}$ extends to a $\mathrm{BT}_{n} E_{n}^{+}$over $Z$ which lifts each $D_{\mathbb{P}_{k, t}^{2}}\left[p^{n}\right]$. For all $n, m \in \mathbb{N}^{*}$, the closed embedding homomorphisms $E_{n, Z \backslash \mathcal{S}_{n+m}} \rightarrow E_{n+m}$ extend to homomorphisms

$$
E_{n}^{+} \rightarrow E_{n+m}^{+}
$$




\section{Purity For BARsotti-TATE GROUPS}

over $Z$ which are closed embeddings that identify $E_{n}^{+}$with $E_{n+m}^{+}\left[p^{n}\right]$, as their restrictions to $\mathbb{P}_{k, 0}^{2}=\mathbb{P}_{k}^{2}$ are so. Thus the inductive system $E_{n}^{+}$is a BT $E^{+}$over $Z$.

The $D_{\mathbb{P}_{k, t}^{2}}$ define a BT $D_{\widehat{Z}}$ over the formal scheme $\widehat{Z}$ of the completion of $Z$ along $\mathbb{P}_{k}^{2}$,

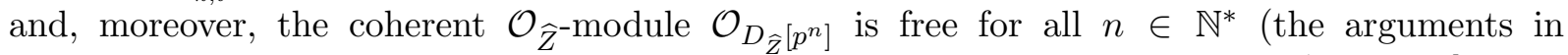
Paragraph 6.1.5 show that the free bases can be chosen compatibly in $t \in \mathbb{N}$ ). From [GD61, Théorème 5.1.4], we get that $D_{\widehat{Z}}$ is the formal completion along $\mathbb{P}_{k}^{2}$ of a uniquely determined (up to unique isomorphism) BT $D_{Z}$ over $Z$ and that for all $n \in \mathbb{N}^{*}$, the coherent $\mathcal{O}_{Z}$-module $\mathcal{O}_{D_{Z}\left[p^{n}\right]}$ is free. Due to the uniqueness part, we have $E^{+}=D_{Z}$.

As the morphism $Z \rightarrow X$ is birational and projective and as $R$ is normal, the ring of global functions on $Z$ is $R$. From this and the fact that for all $n \in \mathbb{N}^{*}$, the coherent $\mathcal{O}_{Z}$-module $\mathcal{O}_{E_{n}^{+}}=\mathcal{O}_{D_{Z}\left[p^{n}\right]}$ is free, we get that $E^{+}=D_{Z}$ is the pullback of a uniquely determined (up to unique isomorphism) BT $D$ over $X$ which extends $D_{U}$ and whose truncations $D\left[p^{n}\right]$ are defined by identities $H^{0}\left(X, D\left[p^{n}\right]\right)=H^{0}\left(Z, E^{+}\left[p^{n}\right]\right.$ ) (to be compared with the Hopf algebra argument involving structure morphisms $\omega_{n}$ at the end of Section 5$)$.

\subsection{The inductive step}

In this subsection, we will assume that $d \geqslant 4$ and that Theorem 1.1(b) holds for regular rings of dimension at most $d-1$. As in Subsection 6.1, for each $d$-tuple $\zeta=\left(\zeta_{1}, \ldots, \zeta_{d}\right) \in W(k)^{d}$ such that its reduction modulo $p$ is not zero, we consider the regular ring $S_{\zeta}:=R /\left(\sum_{i=1}^{d} \zeta_{i} y_{i}\right)$. As $k$ is infinite, from Proposition 3.3 we get that we can choose $\zeta$ such that $S_{\zeta}$ is isomorphic to $W(k)\left[\left[x_{1}, x_{2}, \ldots, x_{d-1}\right]\right] /\left(h_{\zeta}\right)$, where $h_{\zeta} \in W(k)\left[\left[x_{1}, x_{2}, \ldots, x_{d-1}\right]\right]$ is such that its reduction modulo $p$ does not belong to the ideal $\left(x_{1}^{p}, x_{2}^{p}, \ldots, x_{d-1}^{p}\right)+\left(x_{1}, x_{2}, \ldots, x_{d-1}\right)^{2 p-2}$ of $k\left[\left[x_{1}, x_{2}, \ldots, x_{d-1}\right]\right]$. We know that $S_{\zeta}$ is $p$-quasi-healthy by the inductive assumption that Theorem 1.1(b) holds for regular rings of dimension at most $d-1$. Thus $D_{U}$ modulo $\left(\sum_{i=1}^{d} \zeta_{i} y_{i}\right)$ extends to a BT over $\operatorname{Spec}\left(S_{\zeta}\right)$. From this and Lemma 2.8 applied with $\left(R, y, R /(y), D_{U}\right)=\left(R, \sum_{i=1}^{d} \zeta_{i} y_{i}, S_{\zeta}, D_{U}\right)$, we get that $D_{U}$ extends to a BT over $X$. Thus $R$ is $p$-quasi-healthy. This ends the induction and the proof of Theorem 1.1(b).

\section{Proof of Theorem 1.1(a) and Corollary 1.4}

In the situation of Theorem 1.1, from Theorem 1.1(b) and Proposition 3.5, we get that each local ring of $X$ of mixed characteristic $(0, p)$ and of dimension at least 2 is $p$-quasi-healthy. Thus Theorem 1.1(a) is a particular case of the following general lemma whose proof relies on the purity of the branch locus.

Lemma 7.1. Let $Y$ be an integral scheme flat over $\mathbb{Z}$ such that $Y[1 / p]$ is regular and each local ring of $Y$ is normal noetherian. We assume that the following two conditions hold:

(a) Every local ring of $Y$ of mixed characteristic $(0, p)$ and dimension at least 2 is $p$-quasihealthy.

(b) There exists an affine open cover $\left(W_{\lambda}\right)_{\lambda \in \Lambda}$ of $Y$ such that for each $\lambda \in \Lambda$, there exists an $N_{\lambda} \in \mathbb{N}^{*}$ with the property that for every maximal point $\eta_{0} \in Y_{\mathbb{F}_{p}} \cap W_{\lambda}$, the absolute ramification index of $\mathcal{O}_{Y, \eta_{0}}$ is at most $N_{\lambda}$.

If $D_{\eta}$ is a $\mathrm{BT}$ over the generic point $\eta$ of $Y$ which extends to every 1-dimensional local ring of $Y$, then $D_{\eta}$ extends to $Y$ (uniquely up to unique isomorphism).

Proof. As the local rings of $Y$ are normal noetherian, the same arguments as in the proof of 


\section{O. Gabber and A. Vasiu}

[Tat67, Theorem 4] give that the functor

$(\mathrm{BT}$ groups over $Y) \longrightarrow(\mathrm{BT}$ groups over $\eta)$

is fully faithful. Thus it is enough to prove the assertion locally, and therefore we can assume $Y=\operatorname{Spec}(A)=W_{\lambda}($ so $\Lambda=\{\lambda\})$.

In this paragraph, we check that the BT $D_{\eta}$ extends to a BT $D_{Y[1 / p]}$ over the scheme $Y[1 / p]=$ $\operatorname{Spec}(A[1 / p])$. For $n \in \mathbb{N}^{*}$, we consider the normalization $D_{Y[1 / p], n}$ of $Y[1 / p]$ in $D_{\eta}\left[p^{n}\right]$. As the finite étale group scheme $D_{\eta}\left[p^{n}\right]$ extends to a finite étale group scheme over the spectrum of each local ring of $Y[1 / p]$ of dimension 1 (that is, of each local ring of $Y[1 / p]$ which is a discrete valuation ring), the morphism $\vartheta_{n}: D_{Y[1 / p], n} \rightarrow Y[1 / p]$ is finite and étale over each local ring of $Y[1 / p]$ of dimension 1. From this and the purity of the branch locus (see [Gro68, Exposé X, Théorème 3.4(i)]), we get that the morphism $\vartheta_{n}$ is finite and étale over each local ring of $Y[1 / p]$. It is easy to see that this implies that $\vartheta_{n}$ defines a finite étale scheme over $Y[1 / p]$ which extends $D_{\eta}\left[p^{n}\right]$ and thus has a unique group scheme structure which extends the group scheme structure on $D_{\eta}\left[p^{n}\right]$, and this defines a $\mathrm{BT}_{n} D_{Y[1 / p], n}$ over $Y[1 / p]$. For $n, m \in \mathbb{N}^{*}$, the inclusions $D_{\eta}\left[p^{n}\right] \rightarrow D_{\eta}\left[p^{n+m}\right]$ extend to closed embedding homomorphisms $D_{Y[1 / p], n} \rightarrow D_{Y[1 / p], n+m}$, and the inductive system $D_{Y[1 / p], n}$ is the unique (up to unique isomorphism) BT $D_{Y[1 / p]}$ over $Y[1 / p]$ which extends $D_{\eta}$.

To check that $D_{Y[1 / p]}$ extends to $Y$, we consider two cases as follows.

Case 1: $A / p A$ is noetherian. We consider all the local rings $O_{1}, \ldots, O_{t}$ of $R$ which are discrete valuation rings of mixed characteristic $(0, p)$; they correspond to the maximal points $\eta_{1}, \ldots, \eta_{t}$, respectively, of $Y_{\mathbb{F}_{p}}$. For $i \in\{1, \ldots, t\}$, let $D_{O_{i}}$ be the unique (up to unique isomorphism) BT over $\operatorname{Spec}\left(O_{i}\right)$ which extends $D_{\eta}$. For each $n \in \mathbb{N}^{*}$, we consider the largest open subscheme $U_{n}$ of $Y$ which contains $Y[1 / p] \cup\left\{\eta_{1}, \ldots, \eta_{t}\right\}$ and over which there exists a finite flat (locally free) commutative group scheme $D_{U_{n}, n}$ that extends compatibly $D_{Y[1 / p]}\left[p^{n}\right]$ and $D_{O_{i}}\left[p^{n}\right]$ for all indices $i \in\{1, \ldots, t\} .{ }^{4}$ Let $W_{n}$ be the largest open subscheme of $U_{n}$ with the property that the restriction of $D_{U_{n}, n}$ to $W_{n}$ is a $\mathrm{BT}_{n}$ over $W_{n}$. We have a chain of inclusions $W_{1} \supset W_{2} \supset \cdots \supset W_{m} \supset \cdots$. Thus for $Y_{i}:=Y \backslash W_{i}$, we have a chain of inclusions $Y_{1} \subset Y_{2} \subset \cdots \subset Y_{m} \subset \cdots$ between reduced closed subschemes of $Y_{\mathbb{F}_{p}}$. The codimension of each $Y_{m}$ is at least 2. Note that the formation of the open subschemes $U_{n}$ and $W_{n}$ commutes with passage to spectra of local rings of $Y$.

We will show that the assumption that there exists a $q \in \mathbb{N}^{*}$ such that $Y_{q}$ is non-empty leads to a contradiction. We can choose $q \in \mathbb{N}^{*}$ such that for all $m \in \mathbb{N}^{*}$, we have the relations $\operatorname{codim}\left(Y_{q}\right)=\operatorname{codim}\left(Y_{q+m}\right)=c \geqslant 2$.

Let $z$ be a generic point of an irreducible component of $Y_{q}$ of codimension $c$, and let $\tilde{R}:=\mathcal{O}_{Y, z}$. We have $\operatorname{dim}(\tilde{R})=c \geqslant 2$, and therefore $\tilde{R}$ is $p$-quasi-healthy. Let $\tilde{X}:=\operatorname{Spec}(\tilde{R})$, and let $\tilde{U}$ be the punctured spectrum of $\tilde{R}$. From the very definitions and the choice of $z$, we get that $\tilde{U} \cap Y_{n}=\emptyset$ for all $n \in \mathbb{N}^{*}$. Thus there exists a unique (up to unique isomorphism) BT $D_{\tilde{U}}$ over $\tilde{U}$ which extends the restriction of $D_{Y[1 / p]}$ to $\tilde{X}[1 / p]=\operatorname{Spec}(\tilde{R}[1 / p])$. Therefore, $D_{\tilde{U}}$ extends uniquely (up to unique isomorphism) to a BT over $\tilde{X}$, and thus for each $n \in \mathbb{N}^{*}$, we have $z \notin Y_{n}$. This contradicts the fact that $z \in Y_{q}$. Thus our assumption leads to a contradiction. Therefore, for all $n \in \mathbb{N}^{*}$, we have $U_{n}=W_{n}=Y$. This implies that $D_{\eta}$ extends to a BT over $Y$.

\footnotetext{
${ }^{4}$ In the general non-noetherian case, "finite flat" does not imply locally free, but in our case this follows from [GR03, Proposition 2.4.19] applied to classical extensions of the type $A \subset A[1 / p]$.
} 


\section{Purity For BARsotti-TATE GROUPS}

Case 2: general case. Let $n \in \mathbb{N}^{*}$. Let $s \in \mathbb{N}$ be such that it depends only on $N_{\lambda}$ and [Bon06, Theorem E] or [VZ12, Corollary 3] applies to all discrete valuation rings which are local rings of $Y$ at maximal points of $Y_{\mathbb{F}_{p}}$ (see [VZ12, Examples 2 and 4]). Let $z$ be a point of $Y_{\mathbb{F}_{p}}$. From case 1 applied to $\mathcal{O}_{Y, z}$, we get that the restriction of $D_{Y[1 / p]}$ to $\operatorname{Spec}\left(\mathcal{O}_{Y, z}[1 / p]\right)$ extends to a BT $D_{z}$ over $\operatorname{Spec}\left(\mathcal{O}_{Y, z}\right)$. Thus $D_{z}\left[p^{n+s}\right]$ extends to a $\mathrm{BT}_{n+s} E_{n+s, z}$ over an open subscheme $W_{z}$ of $Y$ with $z \in W_{z}$.

From [Bon06, Theorem E] or [VZ12, Corollary 3] and condition (b), we get that for each maximal point $\eta_{0} \in Y_{\mathbb{F}_{p}}$ that belongs to $W_{z}$, the restriction of $E_{n+s, z}\left[p^{n}\right]$ to $\mathcal{O}_{Y, \eta_{0}}$ is (canonically identified with) the truncation of level $n$ of the BT $D_{\eta_{0}}$ over $\mathcal{O}_{Y, \eta_{0}}$ which extends $D_{\eta}$. Based on Fact 2.1(c) applied to the local rings of $Y$, we get that $D_{Y[1 / p]}\left[p^{n}\right]$ and the $E_{n+s, z}\left[p^{n}\right]$ with $z \in Y_{\mathbb{F}_{p}}$ glue together to define a $\mathrm{BT}_{n} D_{n}^{+}$over $Y$ which extends $D_{\eta}\left[p^{n}\right]$ and whose restriction to each $\mathcal{O}_{Y, \eta_{0}}$ is $D_{\eta_{0}}\left[p^{n}\right]$.

If $m \in \mathbb{N}^{*}$, from Fact 2.1(c) applied to local rings of $Y$, we get that the closed embedding homomorphism $D_{Y[1 / p]}\left[p^{n}\right] \rightarrow D_{Y[1 / p]}\left[p^{n+m}\right]$ extends to a homomorphism $D_{n}^{+} \rightarrow D_{n+m}^{+}$. The fact that the inductive system $D_{n}^{+}$is a BT over $Y$ which extends $D_{Y[1 / p]}$ follows from the fact that its restriction to each $\operatorname{Spec}\left(\mathcal{O}_{Y, z}\right)$ is canonically identified with the inductive system $D_{z}\left[p^{n}\right]$. This ends the proofs of the lemma and of Theorem 1.1.

\subsection{Proof of Corollary 1.4}

In the situation of Corollary 1.4(a), condition (a) of Lemma 7.1 holds. Then condition (b) of Lemma 7.1 also holds: for each affine open cover $\left(W_{\lambda}\right)_{\lambda \in \Lambda}$ of $Y$, we can take all $N_{\lambda}$ to be $p-1$; see assertion (ii) of Section 3. Thus Lemma 7.1 implies that Corollary 1.4(a) holds. In view of the uniqueness (up to unique isomorphism) of the extension from the generic point of $Y$, Corollary 1.4(b) is a particular case of Corollary 1.4(a). This ends the proof of Corollary 1.4.

\section{Complements to Corollary 1.4 and Lemma 7.1}

For a topological space $\mathcal{Y}$, let $\mathcal{Y}^{\text {min }}$ be the subspace of maximal points of $\mathcal{Y}$. We recall that if $\mathcal{Y}$ is a locally spectral space (for example, the underlying topological space of a scheme), then $\mathcal{Y}^{\text {min }}$ is retrocompact in $\mathcal{Y}$ if and only if it is pro-constructible, and for $\mathcal{Y}$ spectral, $\mathcal{Y}^{\text {min }}$ is retrocompact in $\mathcal{Y}$ if and only if it is quasi-compact; see [ST10, Corollaries 2.6(i) and 2.7].

For the sake of completeness, for extending BTs from $Y[1 / p]$ to $Y$, we have the following variant of Lemma 7.1 and Corollary 1.4. [In view of [Kol16, Corollary 7], the assumptions on $Y$ imply that for each $z \in Y_{\mathbb{F}_{p}}$, the local ring $\mathcal{O}_{Y, z}$ is either a discrete valuation ring or a noetherian ring of both dimension and depth at least 2 . We will only use this characterization and not the integrally closed condition itself.]

LEMma 8.1. Let $Y$ be scheme on which $p$ is a non-zero-divisor, which is integrally closed in $Y[1 / p]$, and whose local rings at points of residue characteristic $p$ are noetherian. We assume that condition (a) of Lemma 7.1 holds. Then the following two properties hold:

(a) If condition (b) of Lemma 7.1 holds, then each BT $D_{Y[1 / p]}$ over $Y[1 / p]$ which extends at each maximal point of $Y_{\mathbb{F}_{p}}$ extends to a BT over $Y$ (uniquely up to unique isomorphism).

(b) If $Y_{\mathbb{F}_{p}}^{\min }$ is retrocompact (that is, the morphism $Y_{\mathbb{F}_{p}}^{\min } \rightarrow Y$ is quasi-compact), then each BT $D_{W}$ over an open subscheme $W$ of $Y$ that contains $Y[1 / p]$ and $Y_{\mathbb{F}_{p}}^{\min }$ extends to a BT over $Y$ (uniquely up to unique isomorphism); thus, if $Y$ is a faithfully flat regular $\operatorname{Spec}\left(\mathbb{Z}_{(p)}\right)$ scheme, then it is p-healthy regular. 


\section{O. Gabber and A. Vasiu}

Proof. Due to the assumptions of the first sentence of the lemma, the functor

$$
(\text { BT groups over } Y) \longrightarrow(\mathrm{BT} \text { groups over } Y[1 / p])
$$

is fully faithful.

Thus to prove statement (a), we can assume that $Y=W_{\lambda}$ is affine. The remaining part of the proof of statement (a) is similar to cases 1 and 2 of the proof of Lemma 7.1, with just one difference. Namely, unlike in case 2 of the proof of Lemma 7.1, we are not assuming $Y[1 / p]$ regular; once we obtain $E_{n+s, z}$ over an open subscheme $W_{z}$, we have to add that by replacing $W_{z}$ with an affine open subscheme of it (containing $z$ ), we can assume that $E_{n+s, z}[1 / p]$ is isomorphic to the restriction of $D_{Y[1 / p]}\left[p^{n+s}\right]$ to $W_{z}[1 / p]$ under an isomorphism which extends the known isomorphism over $\operatorname{Spec}\left(\mathcal{O}_{Y, z}[1 / p]\right)$.

To prove statement (b), we can assume that $Y$ is quasi-compact and quasi-separated. By the hypotheses, $Y_{\mathbb{F}_{p}}^{\min }$ is quasi-compact. Thus $W$ can be exhausted by quasi-compact open subschemes of it which contain $Y[1 / p] \cup Y_{\mathbb{F}_{p}}^{\min }$, and it is enough to prove the extension assertion for each such open subscheme of $W$. Therefore, we can assume that $W$ is quasi-compact. Let $n \in \mathbb{N}^{*}$. Let $z \in Y_{\mathbb{F}_{p}}$. From statement (a), we get that the restriction of $D_{W}$ to $W \cap \operatorname{Spec}\left(\mathcal{O}_{Y, z}\right)$ extends to a $p$-divisible group $D_{z}$ over $\operatorname{Spec}\left(\mathcal{O}_{Y, z}\right)$. A standard limit argument shows that $D_{z}\left[p^{n}\right]$ spreads out to an extension of $D_{W}\left[p^{n}\right]_{W_{z} \cap W}$ to a BT over $W_{z}$, where $W_{z}$ is an affine open neighborhood of $z$ in $Y$. These extensions are unique (up to unique isomorphism) and glue as we have $\iota_{*}\left(\mathcal{O}_{W}\right)=\mathcal{O}_{Y}$, where $\iota: W \rightarrow Y$ is the open embedding. Thus each $D_{W}\left[p^{n}\right]$ extends to a $\mathrm{BT}_{n}$ over $Y$, and by the uniqueness part, these extensions constitute a BT over $Y$.

Example 8.2. Let $\mathbb{Q} \subset K_{1} \subset K_{2} \subset \cdots$ be a tower of finite field extensions unramified above $p$ such that the union $K_{\infty}:=\cup_{n \in \mathbb{N}^{*}} K_{n}$ has infinitely many places above $p$. Let $O_{n}$ be the integral closure of $\mathbb{Z}_{(p)}$ in $K_{n}$; so $O_{\infty}:=\cup_{n \in \mathbb{N}^{*}} O_{n}$ is the integral closure of $\mathbb{Z}_{(p)}$ in $K_{\infty}$. Let $\mathfrak{m} \in \operatorname{Max}\left(O_{\infty}\right)$ be a non-isolated point. For instance, if $K_{n}:=\mathbb{Q}\left(\sqrt{l_{1}}, \ldots, \sqrt{l_{n}}\right)$, where the $l_{i}$ are distinct primes which are squares in $\mathbb{Q}_{p}$, then $\operatorname{Max}\left(O_{\infty}\right)$ is a Cantor set and so has no isolated point. Let $\pi_{n} \in O_{n}$ be a generator of the product of all maximal ideals of $O_{n}$ different from $\mathfrak{m} \cap O_{n}$. Let $x$ be an indeterminate, and let $A:=\underline{\lim } O_{n}\left[x / \pi_{n}\right]$ (a filtered union of subrings of $O_{\infty}[x / p]$ ). Then the ring $A$ is regular of dimension 2 and $\operatorname{Spec}(A / p A)^{\mathrm{min}}$ is not retrocompact, being a topological space homeomorphic to the disjoint union of $\{\mathfrak{m}\}$ and $\operatorname{Max}\left(O_{\infty}\right) \backslash\{\mathfrak{m}\}$. This example can be modified

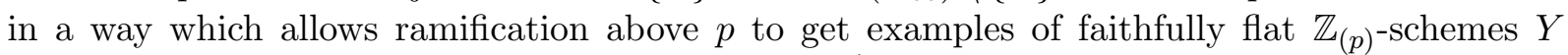
which are regular of dimension 2 and for which $Y^{\text {min }}$ is not retrocompact and condition (b) of Lemma 7.1 does not hold.

Let $R$ be a regular local ring of mixed characteristic $(0, p)$ and dimension $d \geqslant 1$. For $e \in \mathbb{N}^{*}$, we consider the following condition on $R$ :

$\left(\diamond_{e}\right)$ The reduced ring $(R / p R)_{\text {red }}$ is regular, and we have $\operatorname{div}(p)=e \operatorname{div}(p)_{\mathrm{red}}$ (as divisors on $X=\operatorname{Spec}(R))$.

If condition $\left(\diamond_{e}\right)$ holds for $R$, then it also holds for $\widehat{R}$ and for each local ring of $X$ of mixed characteristic $(0, p)$. If $R$ is formally smooth (or only flat with regular special fiber) over a discrete valuation ring $O$ of mixed characteristic $(0, p)$ and absolute ramification index $e$, then condition $\left(\diamond_{e}\right)$ holds for $R$. If $e>1$, there are examples in which condition $\left(\nabla_{e}\right)$ holds for $\widehat{R}$ but not for $R$ and, moreover, $R / p R$ is an integral domain. Moreover, we have the following converse which is related to the applicability of Corollary 1.4 . 


\section{PURITY FOR BARSOTTI-TATE GROUPS}

LEMma 8.3. If condition $\left(\diamond_{e}\right)$ holds for $R$ and $p$ does not divide $e$, then $\widehat{R}$ is a formal power series ring over a complete discrete valuation ring $O$ as above.

Proof. Let $\mathfrak{m}$ be the maximal ideal of $R$; so $k=R / \mathfrak{m}$. We consider a Cohen (coefficient) ring $C(k) \subset \widehat{R}$. As $R$ is a unique factorization domain, from the identity $\operatorname{div}(p)=e \operatorname{div}(p)_{\text {red }}$ of $\operatorname{divisors}$ of $X$ we get that there exist a $\pi \in \widehat{R}$ and a unit $u_{\pi}$ of $\widehat{R}$ such that we have $p=\pi^{e} u_{\pi}$. We write $u_{\pi}=u^{-1} u_{1}$, where $u \in C(k)$ and $u_{1} \in 1+\mathfrak{m} \widehat{R}$. By Hensel's lemma, $u_{1}$ has an $e$ th root $u_{1}^{1 / e}$ in $\widehat{R}$. Thus, by replacing $\pi$ with $\pi u_{1}^{1 / e}$, we can assume that $u_{1}=1$. As $p u=\pi^{e}$, the desired discrete valuation subring of $\widehat{R}$ is $O:=C(k)[\pi]=C(k)\left[x_{d}\right] /\left(x_{d}^{e}-p u\right)$. If $y_{1}, \ldots, y_{d-1} \in \widehat{R}$ lift a regular system of parameters of $(\widehat{R} / p \widehat{R})_{\text {red }}$, it is easy to see that the $C(k)$-algebra homomorphism $C(k)\left[\left[x_{1}, \ldots, x_{d}\right]\right] \rightarrow \widehat{R}$ that maps $x_{i}$ to $y_{i}$ for $i \in\{1, \ldots, d-1\}$ and $x_{d}$ to $\pi$ induces an isomorphism

$$
C(k)\left[\left[x_{1}, \ldots, x_{d}\right]\right]\left(x_{d}^{e}-p u\right)=O\left[\left[x_{1}, \ldots, x_{d-1}\right]\right] \rightarrow \widehat{R} .
$$

\section{ACKNowledGEMEnTS}

The second author would like to thank Binghamton and Bielefeld Universities and I.H.E.S., Bures-sur-Yvette, for good working conditions.

\section{REFERENCES}

Bon06 M.V. Bondarko, The generic fibre of finite group schemes; a "finite wild" criterion for good reduction of abelian varieties, Izv. Math. 70 (2006), no. 4, 661-691; doi:10.1070/ IM2006v070n04ABEH002323.

CCO14 C.-L. Chai, B. Conrad and F. Oort, Complex multiplication and lifting problems, Math. Surveys Mongr., vol. 195 (Amer. Math. Soc., Providence, RI, 2014); doi:10.1090/surv/195.

FC90 G. Faltings and C.-L. Chai, Degeneration of abelian varieties (with an appendix by David Mumford), Ergeb. Math. Grenzgeb. (3), vol. 22 (Springer-Verlag, Berlin, 1990); doi:10.1007/ 978-3-662-02632-8.

GD61 A. Grothendieck and J. Dieudonné, Éléments de géométrie algébrique. III. Étude cohomologique des faisceaux cohérents. I, Publ. Math. Inst. Hautes Études Sci. 11 (1961), no. 1, 5-167; doi: 10.1007/BF02684273.

GD64 Éléments de géométrie algébrique. IV. Étude locale des schémas et des morphismes de schémas. I, Publ. Math. Inst. Hautes Études Sci. 20 (1964), no. 1, 5-251; doi:10.1007/ $\mathrm{BF} 02684747$.

GD67 Éléments de géométrie algébrique. IV. Étude locale des schémas et des morphismes de schémas. IV, Publ. Math. Inst. Hautes Études Sci. 32 (1967), no. 1, 5-333; doi:10.1007/ BF02732123.

GR03 O. Gabber and L. Ramero, Almost ring theory, Lecture Notes in Math., vol. 1800 (SpringerVerlag, Berlin, 2003); doi:10.1007/b10047.

Gro68_ Cohomologie locale des faisceaux cohérents et théorèmes de Lefschetz locaux et globaux (SGA 2), Adv. Stud. Pure Math., vol. 2 (North-Holland Publishing Co., Amsterdam, Masson \& Cie, Éditeur, Paris, 1968).

GV20 O. Gabber and A. Vasiu, The classification of p-quasi-healthy henselian regular rings of dimension 2, manuscript July 2, 2020, available at http://people.math.binghamton.edu/adrian/ GV4.pdf.

Har80 R. Hartshorne, Stable reflexive sheaves, Math. Ann. 254 (1980), no. 2, 121-176; doi:10.1007/ BF01467074. 


\section{O. Gabber and A. Vasiu}

HM68 H. Hironaka and H. Matsumura, Formal functions and formal embeddings, J. Math. Soc. Japan 20 (1968), 52-82; doi:10.2969/jmsj/02010052.

Ill85 L. Illusie, Déformations de groupes de Barsotti-Tate (d'après A. Grothendieck), Astérisque 127 (1985), 151-198.

Kol16 J. Kollár, Variants of normality for Noetherian schemes, Pure Appl. Math. Q. 12 (2016), no. 1, 1-31; doi:10.4310/PAMQ.2016.v12.n1.a1.

LM20 T. Liu and Y.S. Moon, Relative crystalline representations and p-divisible groups in the small ramification case, Algebra Number Theory 14 (2020), no. 10, 2773-2789; doi:10.2140/ant. 2020.14 .2773$.

Mad15 K. Madapusi Pera, The Tate conjecture for K3 surfaces in odd characteristic, Invent. Math. 201 (2015), no. 2, 625-668; doi:10.1007/s00222-014-0557-5.

Mad16 , Integral canonical models for spin Shimura varieties, Compos. Math. 152 (2016), no. 4, 769-824; doi:10.1112/S0010437X1500740X.

Mat89 H. Matsumura, Commutative ring theory, 2nd ed., Cambridge Stud. Adv. Math., vol. 8 (Cambridge Univ. Press, Cambridge, 1989).

Mes72 W. Messing, The crystals associated to Barsotti-Tate groups: with applications to abelian schemes, Lecture Notes in Math., vol. 264 (Springer-Verlag, Berlin - New York, 1972); doi: 10.1007/BFb0058301.

Moo98 B. Moonen, Models of Shimura varieties in mixed characteristics, Galois Representations in Arithmetic Algebraic Geometry (Durham, 1996), London Math. Soc. Lecture Note Ser., vol. 254 (Cambridge Univ. Press, Cambridge, 1998), 267-350; doi:10.1017/CB09780511662010.008.

Moo20 Y.S. Moon, Extending p-divisible groups and Barsotti--Tate deformation ring in the relative case, Int. Math. Res. Not., published online on 02 February 2020, to appear in print; doi: 10.1093/imrn/rnz371.

Ray74 M. Raynaud, Schémas en groupes de type $(p, \ldots, p)$, Bull. Soc. Math. France 102 (1974), 241-280; doi:10.24033/bsmf. 1779.

ST10 N. Schwartz and M. Tressl, Elementary properties of minimal and maximal points in Zariski spectra, J. Algebra 323 (2010), no. 3, 698-728; doi:10.1016/j.jalgebra.2009.11.003.

Tat67 J.T. Tate, p-divisible groups, Proc. Conf. Local Fields (Driebergen, 1966) (Springer, Berlin, 1967), 158-183; doi:10.1007/978-3-642-87942-5_\{\}12.

Vas99 A. Vasiu, Integral canonical models of Shimura varieties of preabelian type, Asian J. Math. 3 (1999), no. 2, 401-518; doi:10.4310/AJM.1999.v3.n2.a8.

Vas04 , A purity theorem for abelian schemes, Michigan Math. J. 52 (2004), no. 1, 71-81; doi: $10.1307 / \mathrm{mmj} / 1080837735$.

Vas06 , Crystalline boundedness principle, Ann. Sci. École Norm. Sup. (4) 39 (2006), no. 2, 245-300; doi:10.1016/j.ansens.2005.12.003.

VZ10 A. Vasiu and T. Zink, Purity results for p-divisible groups and abelian schemes over regular bases of mixed characteristic, Doc. Math. 15 (2010), 571-599.

VZ12__ Boundedness results for finite flat group schemes over discrete valuation rings of mixed characteristic, J. Number Theory 132 (2012), no. 9, 2003-2019; doi:10.1016/j.jnt.2012.03. 010.

ZS58 O. Zariski and P. Samuel, Commutative algebra, Vol. I, Univ. Series in Higher Math. (D. Van Nostrand Co., Inc., Princeton, N.J. - Toronto - London - New York, 1958).

ZS60 - Commutative algebra, Vol. II, Univ. Series in Higher Math. (D. Van Nostrand Co., Inc., Princeton, N.J. - Toronto - London - New York, 1960). 


\section{PURITY FOR BARSOTTI-TATE GROUPS}

Ofer Gabber gabber@ihes.fr

IHÉS, Le Bois-Marie, 35, Route de Chartres, F-91440 Bures-sur-Yvette, France

Adrian Vasiu adrian@math.binghamton.edu

Department of Mathematical Sciences, Binghamton University, Binghamton, P. O. Box 6000, New York 13902-6000, USA 